\title{
Semi-Automatic Control of Network Systems with Non-Monotonic Lyapunov Function
}

\author{
Tri Tran ${ }^{\mathrm{a}}$ and Q. P. Ha ${ }^{\mathrm{b}}$ \\ ${ }^{a}$ Defence Science and Technology Group, Edinburgh, SA 5111, Australia; \\ ${ }^{\mathrm{b}}$ School of Electrical and Data Engineering, Faculty of Engineering and Information \\ Technology, University of Technology Sydney, Australia
}

\begin{abstract}
ARTICLE HISTORY
Compiled August 1, 2019

ABSTRACT

A partially-decentralised scheme for the semi-automatic control of network systems with stabilising agents is presented in this paper. The semi-automatic control of interconnected systems employing the stabilising agent whose installation is segregated from the associated control algorithm has been presented previously. In this development, the quadratic dissipativity constraint (QDC) associated with a non-negative supply rate is newly introduced for the stabilising agent. The closed-loop system having bounded disturbances is input-to-state stabilised with a non-monotonic Lyapunov function when the QDC is used with model predictive control. The effectiveness of the QDC for stabilising agents in the presented partially-decentralised control architecture is demonstrated via simulation studies of a frequency regulation problem in power systems.
\end{abstract}

\section{KEYWORDS}

Stabilising agent; Semi-automatic control; Quadratic dissipativity constraint;

Non-monotonic Lyapunov function.

\section{Introduction}

\subsection{Semi-automatic control and stabilising agent}

In a manufacturing or processing plant, there are always numerous feedback control systems installed to help achieve the operational target with fewer operators. These control systems often have two control modes, automatic and manual, thus from the operation point of view, are semi-automatic. In a semi-automatic control scheme, the control inputs can be from the control law in automatic control mode or the operator in manual control mode. Figure 1 depicts such a semi-automatic control scheme in operation with an automatic controller and a plant operator. This semi-automatic control scheme in the manufacturing and processing plants has not, however, been wide-spread considered in the network systems due to the complex interactions between subsystems.

Among network system applications, the control of power systems and multi-agent systems also has a mix of automatic control and operator manual control at each local

CONTACT Tri Tran. Email: anthony.tran@dst.defence.gov.au; Q. P. Ha. Email: quang.ha@uts.edu.au 


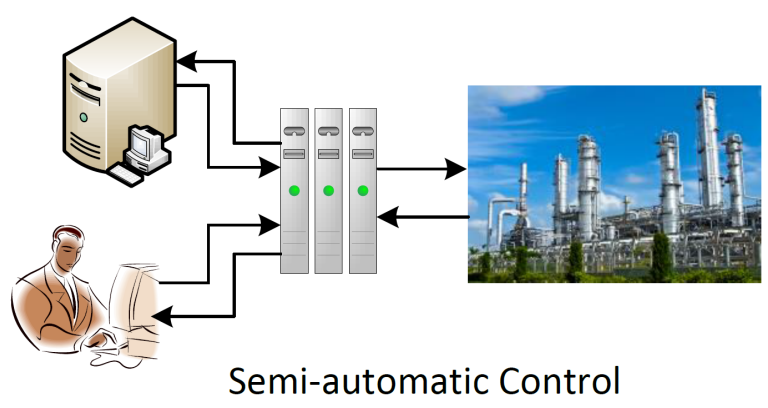

Figure 1. Semi-automatic control with human operator.

subsystem or agent, partly owing to the possibility of communication interruptions or temporary system failures. The semi-automatic control scheme should thus be in place for these applications. This is particularly applicable to human-robot teaming in a consensus or formation control problem, wherein some agents are controlled by human operators while other agents are automatically controlled. The stability guarantee methods for the semi-automatic control scheme have not been addressed in the literature except our previous work Tran, Tuan, Ha, and Nguyen (2011), as of our knowledge.

In this paper, the quadratic dissipativity constraint (QDC) developed previously (Tran, Maciejowski, \& Ling, 2019) is employed in a distributed semi-automatic control scheme for network systems with stabilising agents (SA) (Tran et al., 2011). The method of stabilising network (interconnected) systems with external SA stems from the idea of splitting the stabilising mechanism from the control algorithm, as explained in (Tran \& Ha, 2018b, Chapter 3). The SA is independent to both automatic control and manual control actions. At each time step, the SA determines the stabilising boundary derived from a dissipation-based constraint. The SA subsequently ensures that the control inputs will be within this boundary by overriding (replacing) the control inputs with the corresponding values on the stabilising boundary surface if they are not. As a result, the concept of SA will facilitate the semi-automatic control of network systems.

Quadratic Dissipativity Constraint vs Asymptotically Positive Realness Constraint:

The SA in this work is developed from the QDC presented in (Tran et al., 2019) for systems having disturbances, as an alternative to the Asymptotically Positive Realness Constraint (APRC) presented in (Tran et al., 2011) for systems without disturbances. All the developments are in the discrete-time domain in both of these works. When applying the QDC to Model Predictive Control (MPC), the closed-loop system is input-to-state stabilised with a relaxed non-monotonic Lyapunov function under mild conditions (Tran et al., 2019). The APRC of the following form

$0 \geqslant \xi_{i}\left(k, x_{i}(k), u_{i}(k)\right) \geqslant \gamma_{i}(k) \xi_{i}\left(k-1, x_{i}(k-1), u_{i}(k-1)\right) \forall k \in\left(0, k_{s}\right], 0<\gamma_{i}(k)<1$,

where $\xi_{i}$ is a real-value and time-dependent quadratic function with respect to the state and input pair of $x_{i}$ and $u_{i}$ of a subsystem $\mathcal{S}_{i}$ in an interconnected system $\mathcal{S}$ (i.e. network system) having $h$ subsystems $\mathcal{S}_{i}$, has been used in (Tran et al., 2011) to 
achieve the 'positive realness constraint' status of

$$
\xi_{i}\left(k, x_{i}(k), u_{i}(k)\right) \geqslant 0 \forall k>k_{s}>k_{0}>0,
$$

such that the asymptotic stability is eventually obtained, as a direct result.

In this work, the QDC of the form

$$
0 \geqslant \xi_{i}\left(x_{i}(k), w_{i}(k)\right) \geqslant \beta_{i} \xi_{i}\left(x_{i}(k-1), w_{i}(k-1)\right)-\eta_{i} \quad \forall k>0,0<\beta_{i}<1, \eta_{i}>0,
$$

where $\xi_{i}$ is a real-value, non-positive, and time-independent quadratic function with respect to the state and input pair of $x_{i}$ and $w_{i}=K_{i} x+u_{i}, x=\left[x_{1}, \ldots, x_{h}\right]^{T}$, will be employed for systems having state disturbances, such that

$$
\xi_{i}\left(x_{i}(k), w_{i}(k)\right) \rightarrow-\eta_{i} \text { as } k \rightarrow \infty, \text { and } \xi_{i}\left(x_{i}(k), w_{i}(k)\right) \leqslant 0 \forall k>0 .
$$

The variable $w_{i}$ in the above QDC arises from the virtual perturbed state feedback (PSF) strategy that allows for a limited communication between neighbouring subsystems and fast local computations, firstly introduced in (Tran \& Ha, 2018a). Under this PSF strategy, the control inputs have the virtual form of

$$
u_{i}(k)=w_{i}(k)+\sum_{j} K_{i j} x_{j}(k)=w_{i}(k)+K_{i} x(k)
$$

where $x_{j}$ are the connected neighbours' states, $K_{i j}$ is the state-feedback gain (for clarity, we also call it cooperative-state feedback gain), and $w_{i}$ is the virtual perturbation variable. This is only a virtual PSF form, and will not be applied directly to control the corresponding subsystem. It is used to shape $u_{i}(k)$ that will be computed independently: The MPC optimisation or the plant operator will determine the control $u_{i}(k)$ directly, but not the virtual variable $w_{i}(k)$.

\section{Contributions:}

The application of QDC in a semi-automatic control scheme with SA is presented in this work. This is a novel contribution since the QDC has only been implemented as an enforced stability constraint for the MPC in (Tran et al., 2019). Furthermore, the set-point-independent supply rate function $\xi_{i}\left(\left(x_{i}-\bar{x}_{i}\right),\left(w_{i}-\bar{w}_{i}\right)\right)$ and non-negative storage function $V_{i}\left(x_{i}-\bar{x}_{i}\right)=\left(x_{i}-\bar{x}_{i}\right)^{T} P_{i}\left(x_{i}-\bar{x}_{i}\right), P_{i} \succ 0,\left(\bar{v}_{i}\right.$ is the steady state of $v_{i}$ ) will be adopted for this development together with bounded state disturbances in the system model. In this QDC approach the relaxed Lyapunov function of the closed-loop system is allowed to temporarily increase its value at certain time steps (Tran et al., 2019), and is thus non-monotonic.

In this work, the PSF is used in a partially-decentralised SA scheme with decentralised MPC. The automatic generation control (AGC) problem in power systems has been studied with QDC as an enforced stabilising constraint for the MPC without considering the semi-automatic control requirement in the previous work (Tran \& Ha, 2018a). On the contrary, the SA algorithm is used here instead of MPC stabilising constraints. The semi-automatic control of a network system is thus facilitated for in this development. The resultant control performances with SA have been found compatible with those having QDC integrated into the MPC optimisation as a stabilising constraint in the case study of AGC problem. 


\subsection{Control with non-monotonic Lyapunov function}

Non-monotonic Lyapunov functions have been presented and used previously in the works of Michel, Hou, and Liu (2015) and others, but they had not attracted much attention. Michel et al. (2015) have pointed out that "the roles of the monotonic and the non-monotonic behaviour of Lyapunov functions along system motions were perhaps not fully appreciated" in the past. Partly owing to "the development of the stability and boundedness theory involving non-monotonic Lyapunov functions was incomplete." Only until recently, the non-monotonic Lyapunov functions have started appearing in the developments for switched and hybrid systems, as well as networkedcontrol systems (Chen, Yang, \& Li, 2019; Linsenmayer, Dimarogonas, \& Allgöwer, 2019; Yu \& Wu, 2017). In the work of Yu and Wu (2017), the Lyapunov functions may increase their values during some proper time intervals, and decrease with sufficiently fast rates otherwise. Both discrete-time and continuous-time switched systems have been considered, and the so-called generalized Lyapunov theorems have been derived therein. Discrete-time switched nonlinear time-varying systems have been considered in the work of Chen et al. (2019) together with a weak dissipative-form input-to-state stability (WD-ISS) Lyapunov function having non-monotonic characteristics. As a result, several relaxed stability conditions have been derived for switched systems. Elsewhere, the work of Linsenmayer et al. (2019) has demonstrated how easy it is when non-monotonic Lyapunov functions are employed in the analysis and design of event-triggered strategies for networked-control systems in the discrete-time domain. The stability theorem for discontinuous dynamical systems (DDS) - the terminology used in the work of Michel et al. (2015), has been extended to deal with intermittent data losses. Apart from those, our previous work in (Tran et al., 2019) has presented a general dissipativity constraint (GDC) for the control design of continuous linear and nonlinear systems with disturbances in the discrete-time domain. In that work, the storage function of the closed-loop system is non-monotonic, and the input-to-state stability is obtained under a mild condition of the initial control law continuity. In this work, we apply the GDC in the form of QDC to networked-control systems exploiting the non-monotonic characteristics, such that the stability condition is less conservative. Thus, it is suitable for use with SA that is segregated from the control algorithm.

The remaining of this paper is organised as follows. Notations, system models, problem formulations and the QDC derivation are outlined in Section 2. The nominal stability condition based on the QDC and non-monotonic sotrage function is then given in Section 3. The stability condition for systems having disturbances and suffering from a deterministic data lost process is also stated here. In Section 4, we provide the simulation results for the illustrative example of a four control-area power system with the automatic generation control problem. Section 5 concludes this paper.

\section{Preliminaries and Problem Formulation}

\subsection{Notation}

Capital and lower case letters denote matrices and column vectors, respectively. (.) ${ }^{T}$ denotes the transpose operation. $I_{n}$ and $0_{n}$ denote the $n n$ identity and zero matrices, respectively. $\operatorname{diag}\left[A_{i}\right]_{1}^{N}$ stands for the block-diagonal matrix with diagonal entries $A_{i}, i=1,2, \ldots, N$. $\left\|u_{i}\right\|_{2}$ is the $\ell_{2}-$ norm of vector $u_{i} .\left\|x_{i}\right\|_{\mathcal{Q}}$ is the weighted $\ell_{2}-$ norm 
of $x_{i}, \mathcal{Q} \succ 0 . \lambda_{\max }($.$) indicates the maximum eigenvalue of the argument. \mathbb{R}_{0}^{+}$is the set of non-negative real numbers. A function $\gamma: \mathbb{R}_{0}^{+} \rightarrow \mathbb{R}_{0}^{+}$is called of class $\mathcal{K}$ if it is continuous, strictly increasing and $\gamma(0)=0$. In symmetric block matrices, we use $*$ to denote the off-diagonal symmetric blocks, e.g., $\left[\begin{array}{cc}X & Y \\ * & Z\end{array}\right]=\left[\begin{array}{cc}X & Y \\ Y^{T} & Z\end{array}\right]$. In the discrete time domain, the time index is denoted by $k, k \in \mathbb{Z}$. The bold typeface letters in optimisation formulations are used to emphasise that they are variables.

\subsection{Control System Model}

\subsubsection{Subsystem model}

Consider an interconnected system $\Sigma$ consisting of $h$ nodes representing $h$ subsystems, each denoted as $\mathcal{S}_{i}$, indexed by the elements of the set $\mathcal{N}:=\{1, \ldots, h\}$, and has a discrete-time state space model of the form:

$$
\mathcal{S}_{i}: \quad x_{i}(k+1)=A_{i} x_{i}(k)+B_{i} u_{i}(k)+E_{i} v_{i}(k)+L_{i} d_{i}(k),
$$

where $A_{i} \in \mathbb{R}^{n_{i} \times n_{i}}, B_{i} \in \mathbb{R}^{n_{i} \times m_{i}}, E_{i} \in \mathbb{R}^{n_{i} \times m_{v_{i}}}, L_{i} \in \mathbb{R}^{n_{i} \times q_{i}} ; u_{i} \in \mathbb{R}^{m_{i}}$ and $x_{i} \in \mathbb{R}^{n_{i}}$ are local control and state vectors, respectively; $v_{i} \in \mathbb{R}^{m_{v_{i}}}$ is the interactive, or dynamic coupling, vector; $d_{i} \in \mathbb{R}^{q_{i}}$ is the disturbance vector. The disturbance is unknown but bounded: $\left\|d_{i}\right\|_{2}^{2} \leqslant \theta_{i}$. In the power system applications, the disturbance $d_{i}(k)$ can be a constant, e.g. $d_{i}(k)=d_{c_{i}} \forall k>k_{d_{i}}>0$. For the MPC problem, we consider the state and control constraints: $x_{i} \in \mathbb{X}_{i} \subset \mathbb{R}^{n_{i}}$ and $u_{i} \in \mathbb{U}_{i} \subset \mathbb{R}^{m_{i}}$. The block diagram of $\Sigma$ with decentralised MPC and SA is depicted by Figure 2 .

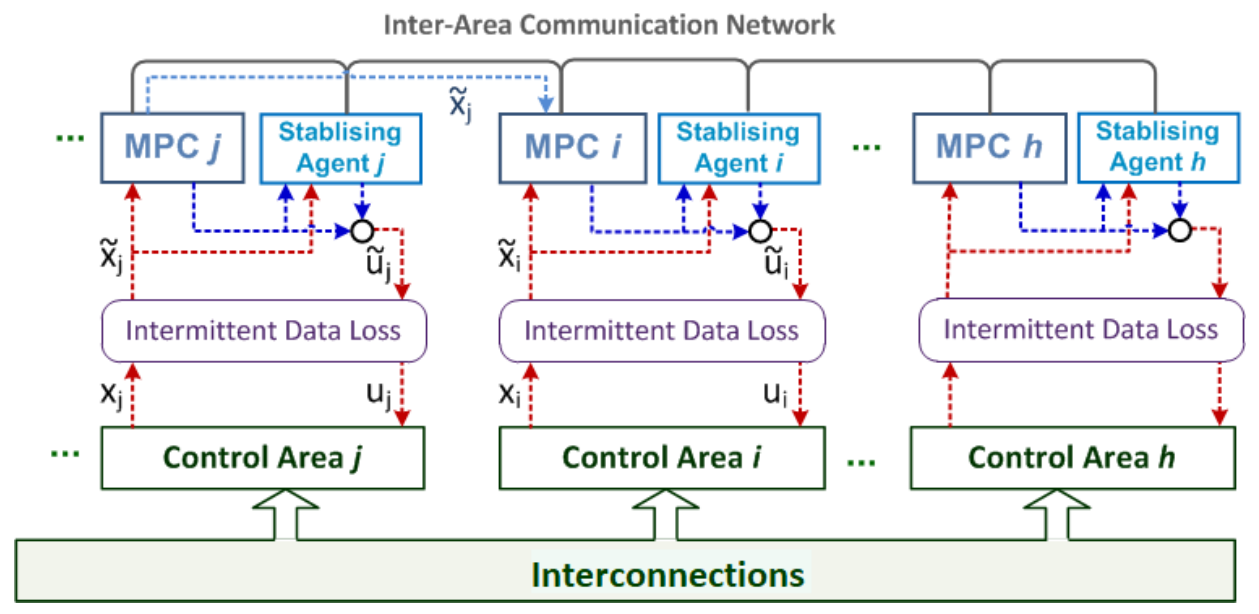

Figure 2. Network system with decentralised MPC and stabilising agents. The inter-area communication network has multiple connection topologies. The inter-area communication channels may become unavailable from time to time.

\subsubsection{Network graph}

A network system is identified by a graph $G=(\mathcal{N}, \mathcal{S}, \mathcal{E})$, where $\mathcal{S}=\left\{\mathcal{S}_{1}, \ldots, \mathcal{S}_{n}\right\}$ is the set of nodes, and $\mathcal{E} \subseteq\left\{\left(\mathcal{S}_{i}, \mathcal{S}_{j}\right): \mathcal{S}_{i}, \mathcal{S}_{j} \in \mathcal{S}, i, j \in \mathcal{N} \mid i \neq j\right\}$ is the set of edges. The graph is undirected (or bidirectional) if for any $i, j \in \mathcal{N},\left(\mathcal{S}_{i}, \mathcal{S}_{j}\right) \in \mathcal{E} \Leftrightarrow\left(\mathcal{S}_{j}, \mathcal{S}_{i}\right) \in \mathcal{E}$. A node $\mathcal{S}_{i} \in \mathcal{N}$ is connected to a node $\mathcal{S}_{j} \in \mathcal{N}$ if there is a path from $\mathcal{S}_{i}$ to $\mathcal{S}_{j}$ in the graph 
following the orientation of the arcs. If $\left(\mathcal{S}_{i}, \mathcal{S}_{j}\right) \in \mathcal{E}$, we say that $\mathcal{S}_{j}$ is neighbor to $\mathcal{S}_{i}$. The set of all neighbors to node $i \in \mathcal{N}$ is denoted as $\mathcal{N}_{i}(G)=\left\{j, j \in \mathcal{N}:\left(\mathcal{S}_{i}, \mathcal{S}_{j}\right) \in \mathcal{E}\right\}$.

Herein, we consider a network of fixed subsystem dynamic couplings, or interconnections, as shown in Figure 2, denoted as $G_{s}=\left(\mathcal{N}, \mathcal{S}, \mathcal{E}_{s}\right)$, and a flexible inter-system communication network, denoted as $G_{c}(k)=\left(\mathcal{N}, \mathcal{S}, \mathcal{E}_{c}\right)$ (the top network in Figure 2). In this work, a set of cooperative-state feedback gains $K_{i j}$ will be determined, each corresponds to a $G_{c}$.

\subsubsection{System model with perturbed state feedback}

Subsystem model is recast with the control $u_{i}(k)=w_{i}(k)+z_{i}(k)$, as follows:

$$
\begin{array}{r}
x_{i}(k+1)=A_{i} x_{i}(k)+B_{i} w_{i}(k)+B_{i} z_{i}(k)+E_{i} v_{i}(k)+L_{i} d_{i}(k), \\
z_{i}(k)=\sum_{j \in \mathcal{N}_{i}\left(G_{c}\right)} K_{i j} x_{j}(k), \quad v_{i}(k)=\sum_{j \in \mathcal{N}_{i}\left(G_{s}\right)} F_{j} x_{j}(k) .
\end{array}
$$

Here, a global coupling matrix $H_{s}$ is used to describe the connection structure of $G_{s}$. The elements of $H_{s}$ are either 1 or 0 only. Similarly, the communication network $G_{c}$ is associated with the matrix $H_{c}$. By denoting the $i^{t h}$ block-row of $H_{s}$ and $H_{c}$, as $H_{s[i]}$ and $H_{c[i]}$, respectively, we have

$$
v_{i}=H_{s[i]} F x, \quad z_{i}=\left[K_{i j}\right]_{[i]} H_{c[i]} x,
$$

where $x:=\left[x_{1}^{T} \ldots x_{h}^{T}\right]^{T}, F:=\operatorname{diag}\left[F_{j}\right]_{1}^{h}$, and $\left[K_{i j}\right]$ is the matrix having the block elements $K_{i j}$.

The global system $\Sigma$ now becomes

$$
\Sigma: x(k+1)=A_{\Sigma} x(k)+B w(k)+L d(k),
$$

where $A_{\Sigma}:=A+E H_{s} F+B K H_{c}, K:=\left[K_{i j}\right]$, in which

$$
\begin{gathered}
A:=\operatorname{diag}\left[A_{i}\right]_{i}^{h}, B:=\operatorname{diag}\left[B_{i}\right]_{i}^{h}, E:=\operatorname{diag}\left[E_{i}\right]_{i}^{h}, L:=\operatorname{diag}\left[L_{i}\right]_{i}^{h}, K:=\left[K_{i j}\right], \\
w=\left[w_{1}^{T} \ldots w_{h}^{T}\right]^{T}, u=\left[u_{1}^{T} \ldots u_{h}^{T}\right]^{T}, d=\left[d_{1}^{T} \ldots d_{h}^{T}\right]^{T}, w \in \mathbb{R}^{m}, u \in \mathbb{R}^{m}, x \in \mathbb{R}^{n} .
\end{gathered}
$$

Assumption 1. (i) All subsystem pairs $\left(A_{i}, B_{i}\right), i=1,2, \ldots, h$, and the global system pairs $(A, B)$ and $\left(A+E H_{s} F, B\right)$ are controllable. (ii) The matrices $I_{n_{i}}-A_{i}, i=$ $1,2, \ldots, h$, and $I_{n}-A-E H_{s} F$ are non-singular. (iii) The updating time instants are synchronised between all subsystems and their controllers.

Assumption 1 is applied throughout this paper.

\subsubsection{Deterministic data losses}

In this development, a sensor/device network is installed for updating the state measurements $\left(x_{i}\right)$ and actuating the control devices $\left(u_{i}\right)$. This is represented by the middle blocks in Figure 2 with "intermittent data loss". The intermittent state and control data loss processes are described as follows:

- The state vector $x_{i}$ of $\mathcal{S}_{i}$ becomes $\tilde{x}_{i}$ beyond the network interface inside the respective controllers. When the state vector $x_{i}(k)$ at the time instant $k$ is trans- 
mitted successfully, we have $\tilde{x}_{i}(k)=x_{i}(k)$, otherwise $\tilde{x}_{i}(k)=x_{i}(k-1)$ (i.e. when the data is lost).

- The intermittent data loss also occurs to the data exchanged between subsystems similarly to $x_{i}(k)$, i.e. $\tilde{x}_{j}(k)$ is received by its neighbors.

- For the control input $\left(u_{i}\right)$, the last successfully received value of $u_{i}$ will be applied to the plant during the data lost time, i.e. $u_{i}(k)=u_{i}(k-1)$, otherwise $u_{i}(k)=$ $\tilde{u}_{i}(k)$.

If we represent the consecutive updating instants of $\tilde{x}_{i}(k), \tilde{x}_{j}(k)$ and $\tilde{u}_{i}(k)$ with a sequence of integer numbers $\mathbb{J}=\left\{j_{1}, \ldots, j_{q}, \ldots, j_{\kappa}, \ldots\right\}$, the time interval between $j_{q}$ and $j_{q+1}$ can be treated as one transmission period. If the communication data is perfect at time $k$, we have $j_{\kappa}=k$. The upper bound of the successful transmission period is denoted as $\mu$ (or MATI - maximum allowable transmission interval).

$$
\mu:=\max _{j_{q} \in \mathbb{J}}(\tau(q)), \quad \tau(q):=j_{q}-j_{q-1} .
$$

The intermittent data losses are accounted for by updating the control $u_{i}$ at every time step - the rolling principle of MPC. This means, the data losses are compensated for in real time, right after each incident, thanks to the MPC together with the QDC that also satisfies the condition of data-lost robust dissipation defined in (11).

\subsection{Quadratic Dissipativity Constraint with Non-zero Set-point}

Denote the set-point-independent input and state vectors as $w_{\triangle i}(k):=w_{i}(k)-$ $\bar{w}_{i}, x_{\triangle i}(k):=x_{i}(k)-\bar{x}_{i}$, where $\bar{x}_{i}$ and $\bar{w}_{i}$ are, respectively, the steady state values of $x_{i}$ and $w_{i}$. Define a dynamic quadratic supply rate in the perfect data environment for node $\mathcal{S}_{i}$, as follows:

$$
\xi_{i}\left(w_{\triangle i}, x_{\triangle i}\right):=w_{\triangle i}^{T} R_{i} w_{\triangle i}+2 x_{\triangle i}^{T} S_{i} w_{\triangle i}+x_{\triangle i}^{T} Q_{i} x_{\triangle i}
$$

where $R_{i}, S_{i}, Q_{i}$ are the coefficient matrices with symmetric $R_{i}$ and $Q_{i}$.

In the deterministic data-lost environment of (6), the supply rates w.r.t. the revived time step $j_{q}$ are considered. For conciseness, $\xi_{i k}\left(x_{\triangle i}\left(j_{q}\right), w_{\triangle i}\left(j_{q}\right)\right)$ is denoted as $\xi_{i(q)}$.

Definition 2.1. The controlled system $\mathcal{S}_{i}(1)$ is said to satisfy the quadratic dissipativity constraint, or simply QDC, if the following inequality holds for all $j_{q} \in \mathbb{J}$ (i.e. for all $k>0)$ :

$$
0 \geqslant \xi_{i(q)} \geqslant \beta_{i} \xi_{i(q-1)}-\epsilon_{i} \theta_{i} .
$$

The small positive number $\epsilon_{i} \theta_{i}$ is added to the QDC in (8) for systems having non-zero disturbances $d_{i}(k) \neq 0,\left\|d_{i}(k)\right\|_{2}^{2} \leqslant \theta_{i}$. When the disturbance vanishes $d_{i}(k)=0$, the nominal QDC will become $0 \geqslant \xi_{i(q)} \geqslant \beta_{i} \xi_{i(q-1)}$, i.e. $\epsilon_{i}=0$.

The right-hand-side inequality can be expressed as an inequality w.r.t $\boldsymbol{u}_{\triangle i}$ when 
the local and respective neighbouring states are known, as follows:

$$
\begin{aligned}
& \boldsymbol{w}_{\triangle i}^{T} R_{i} \boldsymbol{w}_{\triangle i}+2 x_{\triangle i}^{T} S_{i} \boldsymbol{w}_{\triangle i}+x_{\triangle i}^{T} Q_{i} x_{\triangle i}-\beta_{i} \xi_{i(q-1)}+\epsilon_{i} \theta_{i} \geqslant 0 \\
\Leftrightarrow & \boldsymbol{u}_{\triangle i}^{T} R_{i} \boldsymbol{u}_{\triangle i}+2 \Gamma_{i} \boldsymbol{u}_{\triangle i}+\psi_{i} \geqslant 0,
\end{aligned}
$$

where $\Gamma_{i}:=x_{\triangle i}^{T} S_{i}-v_{\triangle i}^{T} K_{i}^{T} R_{i}$,

$$
\psi_{i}:=v_{\triangle i}^{T} K_{i}^{T} R_{i} K_{i} v_{\triangle i}-2 x_{\triangle i}^{T} S_{i} K_{i} v_{\triangle i}+x_{\triangle i}^{T} Q_{i} x_{\triangle i}-\beta_{i} \xi_{i(q-1)}+\epsilon_{i} \theta_{i} .
$$

The non-positive supply rate can be obtained by having $\left[\begin{array}{cc}Q_{i} & S_{i} \\ S_{i}^{T} & R_{i}\end{array}\right] \prec 0$.

The inequality (9) will be adopted by a stabilising agent (SA) in this work to find the stabilising boundary (for $u_{i}$ ) at every time step $j_{q}$, as described in Subsection 1.1. The second condition on the data-lost-robustly dissipative system for (5) is also required here. In this second condition, the global supply rate $\breve{\xi}:=\sum_{i=1}^{h} \breve{\xi}_{i}$, where

$$
\breve{\xi}_{i}\left(x_{\triangle i}, \breve{w}_{\triangle i}\right)=\breve{w}_{\triangle i}^{T} \breve{R}_{i} \breve{w}_{\triangle i}+2 x_{\triangle i}^{T} \breve{S}_{i} \breve{w}_{\triangle i}+x_{\triangle i}^{T} Q_{i} x_{\triangle i}
$$

in which $\breve{R}_{i}:=\operatorname{diag}\left[R_{i}\right]_{1}^{\tau}, \breve{S}_{i}:=\operatorname{diag}\left[S_{i}\right]_{1}^{\tau}, \breve{w}_{\triangle i}:=\left[w_{\triangle i}^{T} w_{\triangle i}^{T} \ldots w_{\triangle i}^{T}\right]^{T}$, is considered

Denote $x_{\triangle}:=\left[x_{\triangle 1}^{T} x_{\triangle 2}^{T} \ldots x_{\triangle h}^{T}\right]^{T}, \breve{d}_{i}:=\left[d_{i}(k-\tau+1)^{T} d_{i}(k-1)^{T} \ldots d_{i}(k)^{T}\right]^{T}$,

$$
\bar{x}:=\left[\begin{array}{llll}
\bar{x}_{1}^{T} & \bar{x}_{2}^{T} \ldots & \bar{x}_{h}^{T}
\end{array}\right]^{T} \text {, and } \bar{w}:=\left[\begin{array}{lll}
\bar{w}_{1}^{T} & \bar{w}_{2}^{T} \ldots & \bar{w}_{h}^{T}
\end{array}\right]^{T} .
$$

Definition 2.2. A controlled system $\Sigma(5)$ subject to the data lost process (6) is said to be data-lost robustly-dissipative w.r.t the global supply rate with disturbance of the form $-\breve{\xi}+\sum_{i=1}^{h} \breve{d}_{i}^{T} \breve{Z}_{i} \breve{d}_{i}$, if there exists a storage function $V\left(x_{\triangle}\right):=x_{\triangle}^{T} P x_{\triangle}, P \succ 0, P$ is full row rank, such that for all controlled motions $(x(k), u(k))$ and steady states $\bar{x}$ and $\bar{u}$, the following dissipation inequality is satisfied irrespectively of the initial state $x(0)$ for all $k>0$ :

$$
\begin{gathered}
V\left(x_{\triangle}(k+\tau)\right)-\sigma V\left(x_{\triangle}(k)\right) \leqslant-\sum_{i=1}^{h}\left[\breve{\xi}_{i}\left(x_{\triangle i}(k), \breve{w}_{\triangle i}(k)\right)-\breve{d}_{i}^{T} \breve{Z}_{i} \breve{d}_{i}\right], \\
\forall \tau \in\{1,2, \ldots, \mu\}, \quad 0<\sigma<1 .
\end{gathered}
$$

Similarly to the traditional dissipative condition with linear matrix inequality (LMI) in the literature, see, e.g., (Brogliato, Lozano, Maschke, \& Egeland, 2006), an LMI can be derived from the dissipation inequality above using the model (5). The problem is firstly stated below.

\subsection{Problem Description}

The task here is:-

(1) To implement the conventional model predictive control (MPC) algorithm (Maciejowski, 2002) using the nominal non-interactive model in the prediction for each single subsystem (node) $\mathcal{S}_{i}(1)$, i.e. the interactive variables $v_{i}$ and $w_{i}$ and disturbance $d_{i}$ vanish in the objective function of each local MPC. The global system may be open-loop unstable. Each node $\mathcal{S}_{i}$ possesses some communication 
channels to its neighbours, but not necessarily with all nodes in $G$. The data dropouts may occur intermittently in these communication channels, as well as in the local state measurements and in the control activations, as defined in (6) above.

(2) To implement a stabilising agent for each $\mathcal{S}_{i}$ to guarantee that the global system $\Sigma$ is asymptotically attractive. In other words, we are concerned with the design of $h$ decoupled stabilising agents associated with the local MPCs of $h$ subsystems $\mathcal{S}_{i}, i=1,2, \ldots, h$, such that the global system $\Sigma(5)$ is asymptotically attractive.

To fulfill the task, the control of the form $u_{i}\left(j_{q}\right)=w_{i}\left(j_{q}\right)+\sum_{j} K_{i j} v_{i}\left(j_{q}\right)$ will be adopted. Here, all nodes in the system must update the available communication links in real time and synchronously apply the updated gains $K_{i j}$ when the communication network changes its connection structure (topology). In the automatic control mode, the control $u_{i}\left(j_{q}\right)$ will be calculated online by the local MPC directly without knowing $w_{i}\left(j_{q}\right)$. In the manual control mode, $u_{i}\left(j_{q}\right)$ will be generated by the operator or unchanged from the previous revived step. To ensure the stability, the control $u_{i}\left(j_{q}\right)$ will be overridden by $\tilde{u}_{i}\left(j_{q}\right)$ generated by the SA once the QDC (9) can not be verified, as described in Subsections 1.1 and 2.5. The decoupled MPC objective function of the following form is considered for each single subsystem (node):

$$
\mathcal{J}_{i}(k)=\sum_{j=0}^{N_{i}}\left\|x_{i}^{T}(k+j)-\bar{x}_{i}\right\|_{\mathcal{Q}_{i}}+\left\|u_{i}^{T}(k+j)-\bar{u}_{i}\right\|_{\mathcal{R}_{i}},
$$

where $\mathcal{Q}_{i}, \mathcal{R}_{i}$ are the weighting matrices (to be chosen by the users), and $N_{i}$ is the predictive horizon. The current local state $x_{i}(k)$ and neighbouring states $x_{j}(k), j \in$ $\mathcal{N}_{i}(G)$, at the time step $k$ (or at the revived time step $j_{q}$, when data are lost) are known. The local optimisation problem of minimising $\mathcal{J}_{i}(k)$ subject to the local model (1), the control constraint $u_{i} \in \mathbb{U}_{i}$ and the local state constraint $x_{i} \in \mathbb{X}_{i}$, in the following:

$$
\min _{\hat{u}} \mathcal{J}_{i}(k)
$$

subject to (1), $v_{i}=0, w_{i}=0, d_{i}=0, u_{i} \in \mathbb{U}_{i}, x_{i} \in \mathbb{X}_{i}$,

is then solved for the minimising sequence $\hat{\boldsymbol{u}}_{i}^{*}$ which consists of $N_{i}+1$ elements of $u_{i}^{*}(k+j), j=0,1, \ldots, N_{i}$. Only the first element $u_{i}^{*}(k)$ is used to control $\mathcal{S}_{i}$. This rolling process is repeated at the next time step, and continues thereon. By virtue of the decoupled objective functions above, the online calculations is computationally less expensive than the other conventional cooperative control schemes with coupled objective functions.

Assumption 2. The $h$ local MPC optimisations (12), $i=1,2, \ldots, h$, are recursively feasible at every reviving time step $j_{q}$; see, e.g., (Mayne, Rawlings, Rao, \& Scokaert, 2000). 


\subsection{Stabilising Agent}

The optimisation problem to determine the stabilising bound $\tilde{\boldsymbol{u}}_{i}$ of a stabilising agent is as follows (Tran et al., 2011):

$$
\begin{gathered}
\min _{\tilde{\boldsymbol{u}}_{i}}\left\|\tilde{\boldsymbol{u}}_{i}-\hat{u}_{i}\right\|^{2}, \\
\text { subject to } \quad \tilde{\boldsymbol{u}}_{\triangle i}^{T} R_{i} \tilde{\boldsymbol{u}}_{\triangle i}+2 \Gamma_{i} \tilde{\boldsymbol{u}}_{\triangle i}+\psi_{i} \geqslant 0 \text { and }\left\|\tilde{\boldsymbol{u}}_{i}\right\|^{2} \in \mathbb{U}_{i},
\end{gathered}
$$

where $R_{i}, \Gamma_{i}, \psi_{i}$ are given in (9).

This means the control $\hat{u}_{i}\left(j_{q}\right)$ from the MPC (or from the operator in the manual control mode) will be overridden by $\tilde{u}_{i}\left(j_{q}\right)$ from the above optimisation whenever the QDC-based inequality (9) is not verified. This means, the stabilising agent will have to verify the satisfaction of the QDC-based inequality (9) at every revived time step $j_{q}$.

\section{Nominal Stability with PSF Strategy}

This section outlines the nominal stability conditions that can be used to determine the cooperative-state feedback gains $K_{i j}$ and the QDC (9). We firstly restate the nominal convergence condition for the global system when there are not any disturbances or data losses.

Lemma 3.1. Consider $\Sigma(5)$ with vanishing $d(k)$, and a non-positive real-value supply rate $\xi(x(k), w(k)), \xi: \mathbb{R}^{n} \times \mathbb{R}^{m} \rightarrow \mathbb{R}_{0}^{+}, \xi(x(0), u(0))$ is finite. Let $\sigma \in \mathbb{R}^{+}, \sigma<1$, and $\beta \in \mathbb{R}^{+}, \beta<1$. Suppose there are two $\mathcal{K}_{\infty}$ functions $\underline{\alpha}(\|x\|), \bar{\alpha}(\|x\|)$ and a real-value non-negative function $V(x(k)), V: \mathbb{R}^{n} \rightarrow \mathbb{R}_{0}^{+}$, such that for each finite $x(0) \in \mathbb{R}^{n}$ the following conditions holds for all $k>0$ :

(1) $\underline{\alpha}(\|x(k)\|) \leq V(x(k)) \leq \bar{\alpha}(\|x(k)\|)$,

(2) $V(x(k))-\sigma V(x(k-1)) \leqslant-\xi(x(k), w(k))$,

(3) $0 \geqslant \xi(x(k), w(k)) \geqslant \beta \xi(x(k-1), w(k-1))$,

with some control sequences $\left\{u(k) \in \mathbb{R}^{m}\right\}$; Then $x(k)$ remains finite and $\|x(k)\| \rightarrow 0$ as $k \rightarrow \infty$.

Proof. In Appendix B.

Next, the robust invariant set (Kerrigan \& Maciejowski, 2000) is defined below.

Definition 3.2. Assuming $u_{i}(k) \in \mathbb{U}_{i}$ and $v_{i}(k) \in \mathbb{V}_{i}$, a set $\mathbb{X}_{i}$ is called robustly constrained control invariant with respect to $\mathbb{U}_{i}$ and $\mathbb{V}_{i}$ for the subsystem (node) $\mathcal{S}_{i}(1)$, nominally, if for all $x_{i}(k) \in \mathbb{X}_{i}$, there exist $u_{i}(k) \in \mathbb{U}_{i}$ such that $x_{i}(k+1) \in \mathbb{X}_{i} \forall k>0$, where $x_{i}(k+1)=A_{i} x_{i}(k)+B_{i} u_{i}(k)+E_{i} v_{i}(k)$ with $u_{i}(k) \in \mathbb{U}_{i}$ and $v_{i}(k) \in \mathbb{V}_{i}$.

Assumption 3. $\mathbb{X}_{i}$ is a robustly constrained control invariant set with respect to $\mathbb{U}_{i}$ and $\mathbb{V}_{i}$ for the subsystem $\mathcal{S}_{i}$ with the PSF (2).

The nominal stability condition for $\Sigma$ with the PSF (5) without any data losses and disturbances is then stated in the next theorem, wherein the global block-diagonal 
matrices $P:=\operatorname{diag}\left[P_{i}\right]_{1}^{h}, Q:=\operatorname{diag}\left[Q_{i}\right]_{1}^{h}, S:=\operatorname{diag}\left[S_{i}\right]_{1}^{h}, R:=\operatorname{diag}\left[R_{i}\right]_{1}^{h}$ are the LMI variables.

Theorem 3.3. Let $0<\sigma<1$. Consider the nominal controlled system $\Sigma$ (5) without any data losses and disturbances, i.e $\mathbb{J}=\mathbb{Z}^{+}$and $d_{i}(k)=0$, that have the controls $u_{i}(k)=u_{i}^{*}(k)$ from the decentralised MPC optimisations (12), or taken the values from the respective operators, $i=1,2, \ldots, h$. Suppose that

(1) Assumptions 3 holds true;

(2) The following LMIs are feasible:

$$
\begin{gathered}
{\left[\begin{array}{cccc}
\boldsymbol{P} & \boldsymbol{P} A_{\Sigma} & \boldsymbol{P} B & -\boldsymbol{P B} \\
* & \sigma \boldsymbol{P}-\boldsymbol{Q} & -\boldsymbol{S} & \boldsymbol{S}+(\sigma \boldsymbol{P}-\boldsymbol{Q}) \mathcal{B} \\
* & * & -\boldsymbol{R} & \boldsymbol{R}+\boldsymbol{S}^{T} \mathcal{B} \\
* & * & * & \mathcal{B}^{T}(\sigma \boldsymbol{P}-\boldsymbol{Q}) \mathcal{B}-2 \mathcal{B}^{T} \boldsymbol{S}-\boldsymbol{R}
\end{array}\right] \succ 0,} \\
\\
{\left[\begin{array}{cc}
\boldsymbol{Q}_{i} & \boldsymbol{S}_{i} \\
\boldsymbol{S}_{i}^{T} & \boldsymbol{R}_{i}
\end{array}\right] \prec 0, \quad i=1, \ldots h,}
\end{gathered}
$$

where $\mathcal{B}:=\left(I_{n}-A\right)^{-1} B$ and $A_{\Sigma}=A+E H_{s} F+B K H_{c}$;

(3) (a) $u_{i}(k)=\tilde{u}_{i}(k)$ if the control $u_{i}(k)$ does not verify (9), where $\tilde{u}_{i}(k)$ is a solution to (13),

(b) $x_{i}(k+1) \in \mathbb{X}_{i} \forall k>0, x_{i}(k+1)=A_{i} x_{i}(k)+B_{i} \tilde{u}_{i}(k)+E_{i} v_{i}(k)$,

$$
i=1, \ldots, h
$$

Then the nominal system $\Sigma(5)$ is guaranteed stabilised by $h$ stabilising agents in the sense that $x(k)$ remains bounded and $x(k) \rightarrow 0$ as $k \rightarrow \infty$.

Proof. In Appendix B.

Remark 1. The condition (4) in Theorem 3.3 is required for the recursive feasibility of MPC since the optimisation of the stabilising agent in (13) does not (and can not) include the state constraint in the problem formulation. Nevertheless, this has not been found an issue with the AGC problem in the simulation studies.

In order to solve the matrix inequality (14) in Theorem 3.3 and determine the gains $K_{i j}$ of the form $K_{i j}=Y_{i j} P$, similarly to the development in (Kothare, Balakrishnan, \& Morari, 1996), the following LMI is employed instead of (14):

$$
\left[\begin{array}{cccc}
\mathcal{P} & \left(A+E H_{s} F\right) \mathcal{P}+B \boldsymbol{Y} & B \mathcal{P} & -\mathcal{B P} \\
* & \boldsymbol{M} & -\boldsymbol{S} & \boldsymbol{S}+\boldsymbol{M B} \\
* & * & -\boldsymbol{R} & \boldsymbol{R}+\boldsymbol{S}^{T} \mathcal{B} \\
* & * & * & \mathcal{B}^{T} \boldsymbol{M B}-2 \mathcal{B}^{T} \boldsymbol{S}-\boldsymbol{R}
\end{array}\right] \succ 0,
$$


where $\mathcal{P}:=\boldsymbol{P}^{-1}, \boldsymbol{M}:=\sigma \boldsymbol{P}-\boldsymbol{Q}, \boldsymbol{Y}:=\operatorname{diag}\left[\sum_{j} \boldsymbol{Y}_{i j}\right]_{1}^{h}$. The matrix $\boldsymbol{Q}$ can be recovered from $\boldsymbol{P}=\mathcal{P}^{-1}$ and $\boldsymbol{M}$. With the zero steady states, the above LMI becomes

$$
\left[\begin{array}{ccc}
\mathcal{P} & \left(A+E H_{s} F\right) \mathcal{P}+B \boldsymbol{Y} & B \mathcal{P} \\
* & \boldsymbol{M} & -\boldsymbol{S} \\
* & * & -\boldsymbol{R}
\end{array}\right] \succ 0 .
$$

The following theorem states the stability condition for $\Sigma$ when there are intermittent data losses and state disturbances.

Theorem 3.4. Let $0<\sigma<1$. Consider the controlled system $\Sigma(5)$ with the data lost process (6) and non-zero disturbance $d_{i}(k) \neq 0$, that have the controls $u_{i}(k)=u_{i}^{*}(k)$ from the decentralised MPC optimisations (12), or taken the values from the respective operators, $i=1,2, \ldots, h$. Suppose that

(1) Assumptions 3 holds true;

(2) The dissipation inequality (11) is fulfilled;

(3) (a) The supply rate $\xi_{i}\left(x_{\triangle i}, w_{\triangle i}\right)$ (7) is non-positive,

(b) $u_{i}(k)=\tilde{u}_{i}(k)$ if the control $u_{i}(k)$ does not verify (9), where $\tilde{u}_{i}(k)$ is a solution to (13),

(c) $x_{i}(k+1) \in \mathbb{X}_{i} \forall k>0, x_{i}(k+1)=A_{i} x_{i}(k)+B_{i} \tilde{u}_{i}(k)+E_{i} v_{i}(k)$,

$$
i=1, \ldots, h
$$

Then the controlled system $\Sigma(5)$ is guaranteed stabilised by $h$ stabilising agents in the sense that $x(k)$ remains bounded and $x(k)$ converges to a ball centred on 0 whose radius is a $\mathcal{K}$-function of $\rho$, where $\rho>0$ depends on $\theta_{i}$ and $\epsilon_{i}, i \in\{1,2, \ldots, h\}$.

Proof. In Appendix B.

The LMI dissipative condition in (2) of Theorem 3.4 is as follows:

$$
\left[\begin{array}{ccccc}
\boldsymbol{P} & \boldsymbol{P} \mathscr{A} & \boldsymbol{P} \mathscr{B} & -\boldsymbol{P} \mathscr{B}_{A} & -\boldsymbol{P}\left(\mathscr{L}-\mathscr{L}_{A}\right) \\
* & \sigma \boldsymbol{P}-\boldsymbol{Q}_{\tau} & -\boldsymbol{S}_{\tau} & \boldsymbol{S}_{\tau}+\left(\sigma \boldsymbol{P}-\boldsymbol{Q}_{\tau}\right) \mathscr{B}_{A} & \boldsymbol{Q}_{\tau} \mathscr{L}_{A} \\
* & * & -\boldsymbol{R}_{\tau} & \boldsymbol{R}_{\tau}+\boldsymbol{S}_{\tau}^{T} \mathscr{B}_{A} & \boldsymbol{S}_{\tau}^{T} \mathscr{L}_{A} \\
* & * & * & \mathscr{B}_{A}^{T}\left(\sigma \boldsymbol{P}-\boldsymbol{Q}_{\tau}\right) \mathscr{B}_{A}-2 \mathscr{B}_{A}^{T} \boldsymbol{S}_{\tau}-\boldsymbol{R}_{\tau} & \left(\boldsymbol{S}_{\tau}^{T}-\mathscr{B}_{A}^{T} \boldsymbol{Q}_{\tau}\right) \mathscr{L}_{A} \\
* & * & * & * & \boldsymbol{Z}_{\tau}-\mathscr{L}_{A}^{T} \boldsymbol{Q}_{\tau} \mathscr{L}_{A}
\end{array}\right] \succ 0,
$$

where

$$
\begin{aligned}
& \mathscr{B}:=\left[\begin{array}{lllll}
\left(A_{\Sigma}\right)^{\tau-1} B & \left(A_{\Sigma}\right)^{\tau-2} B & \ldots & A_{\Sigma} B & B
\end{array}\right], \\
& \mathscr{L}:=\left[\begin{array}{llll}
\left(A_{\Sigma}\right)^{\tau-1} L & \left(A_{\Sigma}\right)^{\tau-2} L \ldots A_{\Sigma} L & L
\end{array}\right], \\
& \mathscr{A}:=\left(A_{\Sigma}\right)^{\tau}, \mathscr{B}_{A}:=(I-\mathscr{A})^{-1} \mathscr{B}, \mathscr{L}_{A}:=(I-\mathscr{A})^{-1} \mathscr{L}, \\
& \boldsymbol{Q}_{\tau}:=\operatorname{diag}\left[\boldsymbol{Q}_{i}\right]_{1}^{\tau}, \boldsymbol{S}_{\tau}:=\operatorname{diag}\left[\breve{\boldsymbol{S}}_{i}\right]_{1}^{\tau}, \boldsymbol{R}_{\tau}:=\operatorname{diag}\left[\breve{\boldsymbol{R}}_{i}\right]_{1}^{\tau}, \boldsymbol{Z}_{\tau}:=\operatorname{diag}\left[\breve{\boldsymbol{Z}}_{i}\right]_{1}^{\tau} .
\end{aligned}
$$


It is derived by substituting the model of $\Sigma(5)$ and the predictive state vector of the form $x(k+\tau)=\mathscr{A} x(k)+\mathscr{B} \breve{w}(k)+\mathscr{L} \breve{d}(k)$, and the steady state $\bar{x}=(I-\mathscr{A})^{-1} \mathscr{B} \breve{w}+(I-\mathscr{A})^{-1} \mathscr{L} \breve{d}(k)$, into the dissipation inequality (11), then rearranging the matrices to form the inequality in the variables $x(k), \breve{w}(k), \breve{w}$, and $\breve{d}(k)$.

We will firstly determine the feasible $Q_{i}, S_{i}, R_{i}, P_{i}$, and $K_{i j}$ from the LMI (16), then re-use the resultant $R_{i}$ and $P_{i}$ to determine $S_{i}$ and $Q_{i}$ from the LMI (18) having only $S_{i}, Q_{i}$ and $Z_{i}$ as variable, as follows:

$$
\left[\begin{array}{ccccc}
P & P \mathscr{A} & P \mathscr{B} & -P \mathscr{B}_{A} & -P\left(\mathscr{L}-\mathscr{L}_{A}\right) \\
* & \sigma P-\boldsymbol{Q}_{\tau} & -\boldsymbol{S}_{\tau} & \boldsymbol{S}_{\tau}+\left(\sigma P-\boldsymbol{Q}_{\tau}\right) \mathscr{B}_{A} & \boldsymbol{Q}_{\tau} \mathscr{L}_{A} \\
* & * & -R_{\tau} & R_{\tau}+\boldsymbol{S}_{\tau}^{T} \mathscr{B}_{A} & \boldsymbol{S}_{\tau}^{T} \mathscr{L}_{A} \\
* & * & * & \mathscr{B}_{A}^{T}\left(\sigma P-\boldsymbol{Q}_{\tau}\right) \mathscr{B}_{A}-2 \mathscr{B}_{A}^{T} \boldsymbol{S}_{\tau}-R_{\tau} & \left(\boldsymbol{S}_{\tau}^{T}-\mathscr{B}_{A}^{T} \boldsymbol{Q}_{\tau}\right) \mathscr{L}_{A} \\
* & * & * & * & \boldsymbol{Z}_{\tau}-\mathscr{L}_{A}^{T} \boldsymbol{Q}_{\tau} \mathscr{L}_{A}
\end{array}\right] \succ 0,
$$

The control algorithm with the PSF for each single subsystem consisting of a local MPC, an operator, and a stabilising agent is as follows:

Procedure 1. - Partially Decentralised Semi-Automatic Control with MPC and PSF

(1) Off-line: Select the parameters $\sigma, \beta_{i}$, and $\epsilon_{i}, i=1,2, \ldots, h$. Determine the QDC coefficient matrices $Q_{i}, S_{i}, R_{i}$ and the cooperative-state feedback gains $K_{i j}$ for all possible connection structures $G_{c}$ (with different $H_{c}$ ) from LMIs (16) and (19).

(2) Online: At every revived time step $j_{q}>0$,

(a) Solve the MPC optimisation (12) for the minimising vector $u_{i}^{*}(k)$ using the known local state $x_{i}\left(j_{q}\right)$.

(b) Send the value $u_{i}^{*}(k)$ to the stabilising agent.

(c) The stabilising agent then

(i) Receives the control $\hat{u}_{i}(k)$ from the MPC (i.e. $\left.\hat{u}_{i}(k)=u_{i}^{*}(k)\right)$ in the automatic control mode or from the operator in the manual control mode.

(ii) Receives the neighbouring states $x_{j}\left(j_{q}\right)$ via the available communication channels.

(iii) Updates the QDC inequality (9) using the calculated $x_{\triangle i}\left(j_{q}\right), \xi_{i(q-1)}$, and the local states $x_{i}\left(j_{q}\right)$, as well as the off-line computed coefficient matrices $Q_{i}, S_{i}, R_{i}$, and the respective gains $K_{i j}$.

(iv) Verifies the satisfaction of $(9)$ with $\hat{u}_{i}(k)$.

- If (9) holds true, outputs the control $\hat{u}_{i}(k)$ to manipulate the subsystem $\mathcal{S}_{i}$.

- Otherwise solves the optimisation (13) for $\tilde{u}_{i}$, then overrides $\hat{u}_{i}(k)$ with $\tilde{u}_{i}$ and outputs the control $\tilde{u}_{i}$ to manipulate the subsystem $\mathcal{S}_{i}$.

(d) Return to the first online step a).

Numerical simulation with the AGC problem for a power system having four control areas is studied in Matlab R2015b environment with Yalmip toolbox and SeDuMi v1.3 SDP solver in the next section. 


\section{Illustrative Example}

\subsection{Automatic Generation Control - A Power System Application}

Power systems are typical examples of interconnected systems whose subsystems are dynamically coupled. Among many alternating current (AC) power system applications, the 'automatic generation control' problem (Wood, Woolenberg, \& Sheblé, 2013), in which a large-scale power system is formed by interconnected subsystems called 'control areas', has been identified as the key participant in the secondary control layer to regulate the electrical frequency and the 'tie-line' power flows (linearly approximated power flows) between the control areas.

In a traditional $\mathrm{AC}$ power system, the electrical frequency of a synchronous generator is regulated to maintain a near constant nominal value of $50 \mathrm{~Hz}$ or $60 \mathrm{~Hz}$. For a turbine-generation set (power generation source), a 'governor' is installed in a local control panel for this task. The governor is a controller in a feedback control loop with the frequency as a controlled variable. For a multiple-machine power system (multiple power generation sources), such local governors will not be able to eliminate the steady-state offset values, in other words, deviations from the nominal values in the steady state, when there are non-negligible permanent load changes.

The current technique to solve this problem, i.e. to bring the frequency back to the nominal value within acceptable deviations, is to adjust the output powers of generators at respective local control areas. Such adjustments can be computed from the small-signal model (with incremental variables) that also includes the tie-line power flows between the allocated control areas and the mechanical powers required from the corresponding prime-movers - the turbines. A centralised control scheme is often the norm for this problem which is usually referred to as 'automatic generation control' (Wood et al., 2013) problem representing a conventional multi-variable supplement frequency control scheme. It is called 'supplement' because the primary frequency regulation is the responsibility of the local governor.

The AGC problem with small-signal models of a power system defines a multivariable control problem of a state-coupled control-decoupled interconnected system as in the control literature, see, e.g. (Lunze, 1992). Furthermore, there is always an option for manual manipulations of a local subsystem in this AGC application. Therefore, the SA algorithm that is segregated from the control algorithm for guaranteeing the global system stability is a suitable solution for this application.

It is worth noting here that, AGC is a classical problem in power systems. There are currently progressive research works on new control problems in power systems that integrate sustainable energy sources and smart grids, see e.g. (Weitenberg et al., 2018), and this is outside the scope of this illustrative example. The semi-automatic control scheme with stabilising agents is, nevertheless, applicable to generic network systems described in Section 2.

\subsection{Case studies}

The small-signal model without local losses is treated as a linear state-space model for the multi-variable control design, similarly to the work in (Tran \& Ha, 2018a). The (global) open-loop system is unstable. The simulation studies here have re-used the model parameters and settings in (Tran \& Ha, 2018a), as follows (and Appendix A):

Sampling and updating time: $T_{s}=2$. Initial state vectors: $x_{1}=0 ; x_{2}=0 ; x_{3}=$ $0 ; x_{4}=0$. State constraint: $(-\infty,+\infty)$. Control constraint: $\left|u_{i}(k)\right| \leqslant 0.5$. Weighting 


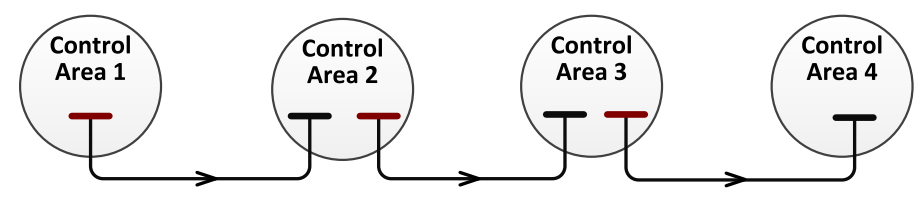

Figure 3. Control areas are serially connected via tie-lines (Tran \& Ha, 2018a).

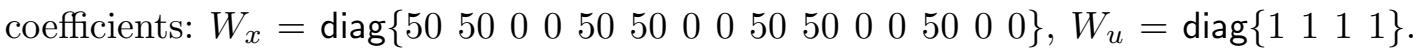
Predictive horizon: $N=4$. Permanent (constant) load disturbance: In this simulation study, the load in area 2 increases $25 \%$ while the load in area 3 decreases $25 \%$ from the time step 2 onward $\left(\Delta P_{L}\right)$. The weighting coefficients for the two state variables $\Delta P_{\text {mech }}$ and $\Delta Z$ are set to 0 . The trajectories of state and control elements from the centralised MPC are shown in Figure 4a.

\subsection{Simulation results without the PSF strategy in a perfect data environment}

Case study 1a:

The centralised MPC with the predictive horizon of $N=12$ provides a good performance for this example. However, the centralised MPC with $N=4$ is unstable without the stabilising agent as shown in Figure 4b. 

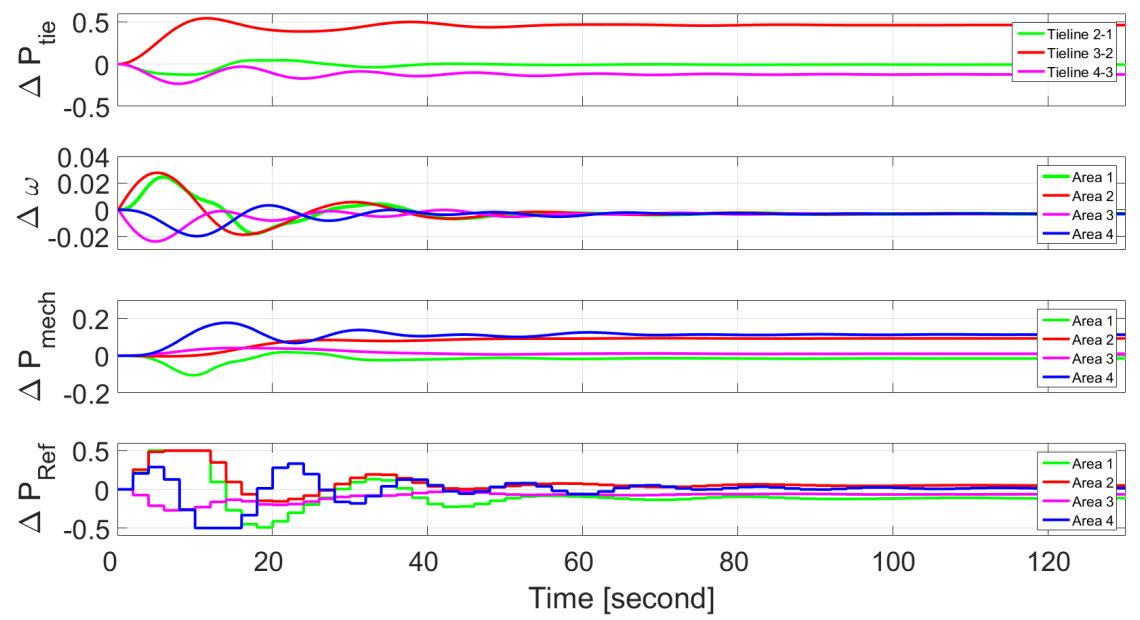

(a) With a longer predictive horizon $\mathrm{N}=12$. The steady states are reached after 70 seconds, approximately.
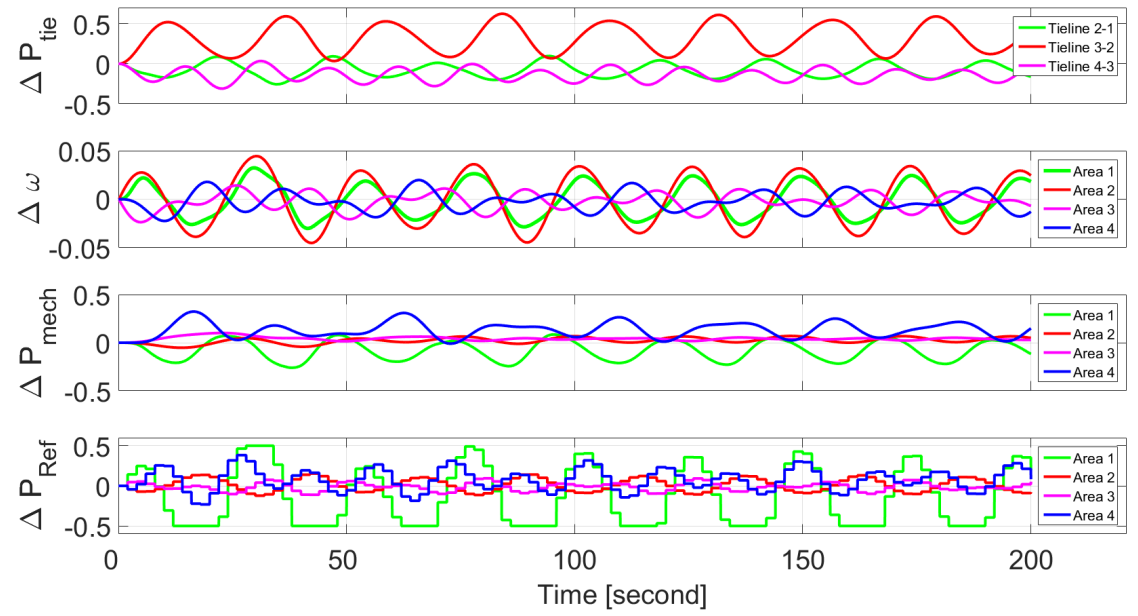

(b) With a shorter predictive horizon $\mathrm{N}=4$. The system is not stabilised.

Figure 4. Centralised MPC without the stabilising agent.

Case study 1b:

In a fully-decentralised MPC (DeMPC) that uses the same predictive horizons of $N=4$ for all subsystems, the system is not unstable, but the control performance is much worse than that in the centralised MPC with $N=12$. When the DeMPC is implemented with SA developed in this paper using Procedure 1 and $N=4$, the time responses in Figure 5 show a control performance comparable to the centralised MPC having a longer predictive horizon $N=12$, as in Figure 4a. The settling time with DeMPC is, however, $25 \%$ longer, approximately. 

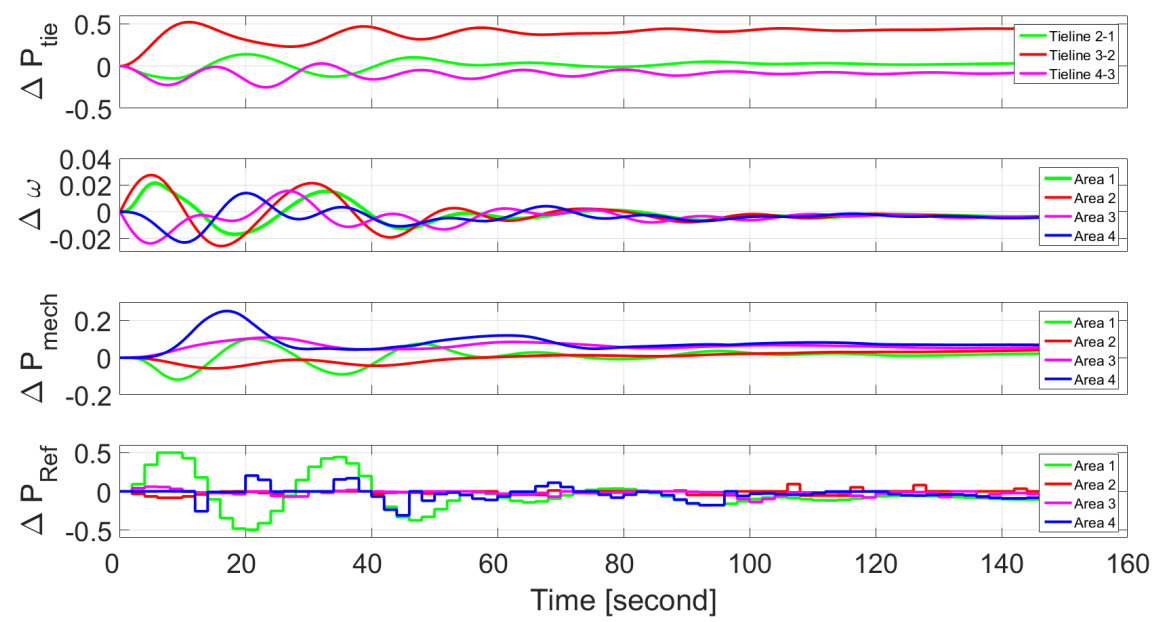

Figure 5. Fully decentralised MPC with stabilising agent and a predictive horizon $\mathrm{N}=4$. The control performance is comparable to that of the centralised MPC with $N=12$ in Figure 4a. The steady states are reached after 120 seconds, approximately, which is longer than that in the centralised MPC.

\subsection{Simulation results with the PSF strategy in a perfect data environment}

Case study 2:

We now implement the PSF strategy with cooperative-state feedback gains to this AGC problem. The control performances of the DeMPC with PSF and SA have been improved with the smaller steady states of tie-line power flows in these two case studies, one with the full cooperative states from all neighboring control areas in Figure 6a (case study 2a), and the other with only a part of the cooperative states in Figure $6 \mathrm{~b}$ (case study $2 \mathrm{~b}$ ).

In Figure 6a, the cooperative states are from the control area $2 \rightarrow 1,1 \rightarrow 2,3 \rightarrow 2$, $2 \rightarrow 3,4 \rightarrow 3$, and $3 \rightarrow 4$. In Figure $6 \mathrm{~b}$, the cooperative states are from the control areas $2 \rightarrow 1,1 \rightarrow 2,3 \rightarrow 2$, and $3 \rightarrow 4$ only, i.e. two communication links are missing.

The tie-line power steady states with partial cooperative states in case study $2 \mathrm{~b}$ are similar to those with full cooperative states in case study $2 \mathrm{a}$, but the control moves are slightly larger. They are much smaller than those in the DeMPC of case study $1 \mathrm{~b}$ without any cooperative state feedbacks shown in Figure 5. 

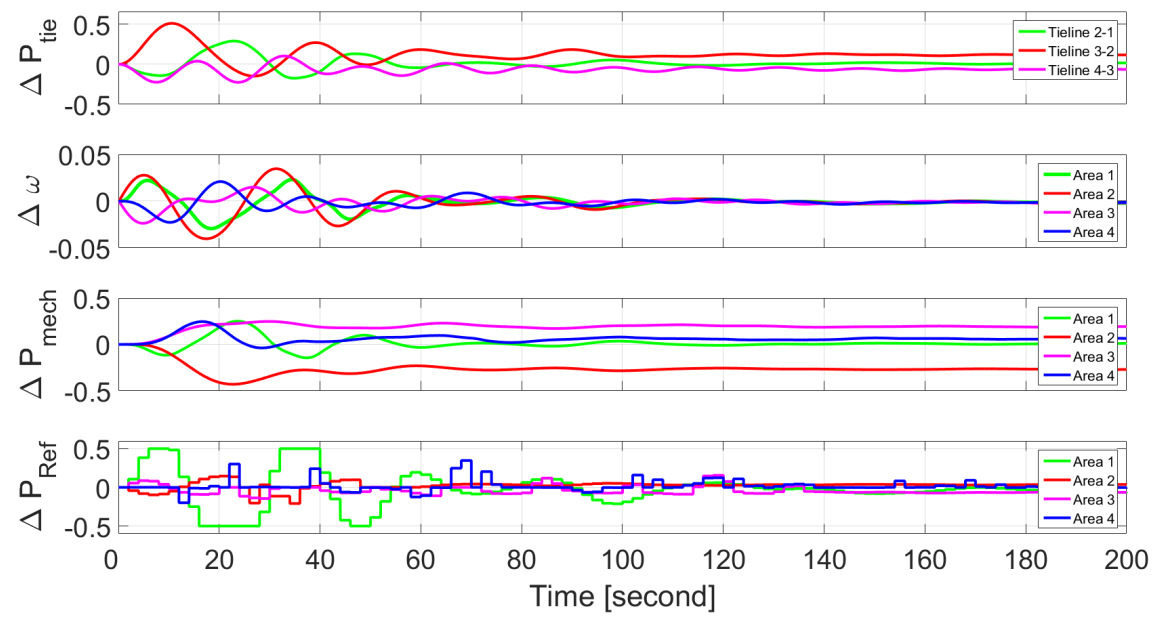

(a) Case study 2a - Full cooperative states. The steady states are reached after 140 seconds, approximately. The tie-line power steady states shown in the top sub-figure are much smaller than those in the DeMPC in Figure 5.
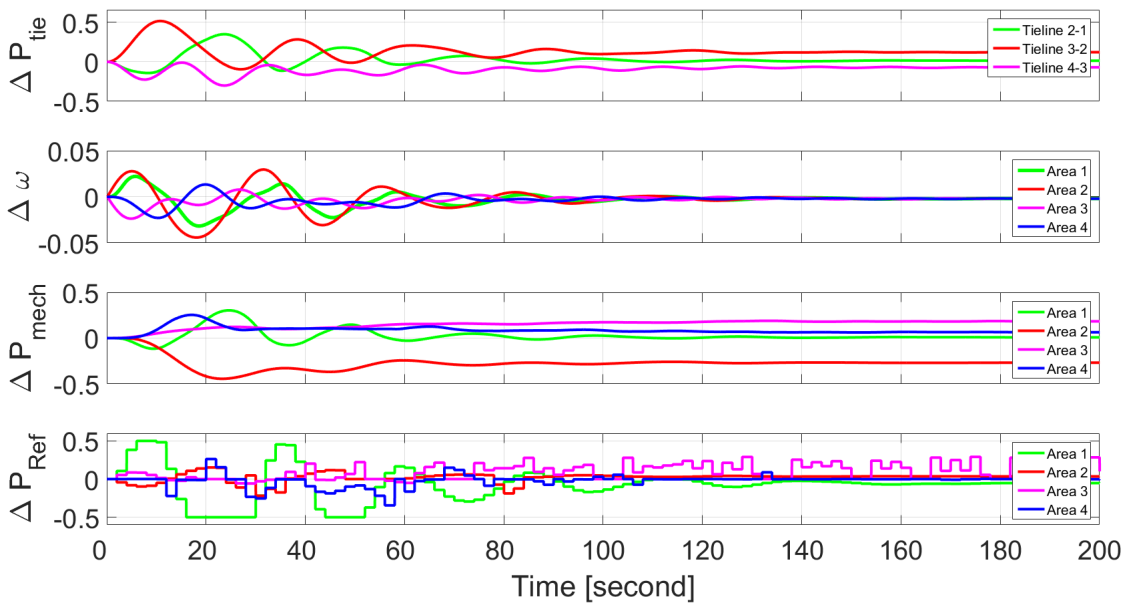

(b) Case study 2b - Partial cooperative states. The steady states are also reached after 140 seconds, approximately. Two communication links (to the control area no. 3) are missing. The tie-line power steady states are still similar to those with full cooperative states in case $2 \mathrm{a}$ above, but the control moves are slightly larger.

Figure 6. Decentralised MPC with PSF and stabilising agent, predictive horizon $\mathrm{N}=4$ - Two case studies with full and partial cooperative states. The tie-line power steady states are much smaller than those in the DeMPC without any cooperative state feedbacks shown in Figure 5.

Case study 3:

Now, we assume that only two communication links are available in the next case study: The cooperative states are from the control areas $2 \rightarrow 1$ and $3 \rightarrow 4$ only, i.e. four communication links are missing. The resulting time responses are shown in Figure $7 \mathrm{a}$.

Case study 4:

In this fourth case study, two communication links are missing similarly to the case study $2 \mathrm{~b}$, but they are different links. The cooperative states are from the control areas $2 \rightarrow 1,2 \rightarrow 3,4 \rightarrow 3$, and $3 \rightarrow 4$. The resulting time responses are shown in Figure $7 \mathrm{~b}$. 
The performances in both case studies 3 and 4 in Figures $7 \mathrm{a}$ and $7 \mathrm{~b}$ are briefly summarised as follows: The tie-line power steady states and the settling times are slightly worse than those in the case study $2 \mathrm{a}$ with full cooperative states, but are better than those in case studies $1 \mathrm{~b}$ without any cooperative states shown in Figures 5 . Notably, they are also slightly worse than those in the case study $2 \mathrm{~b}$ with a different set of partial cooperative states. The control moves in case study 4 are, however, smaller than those in case study 3 . This can be explained by comparing the number of communication links available: four communication links are missing in case study 3 while only two are missing in case study 4 .
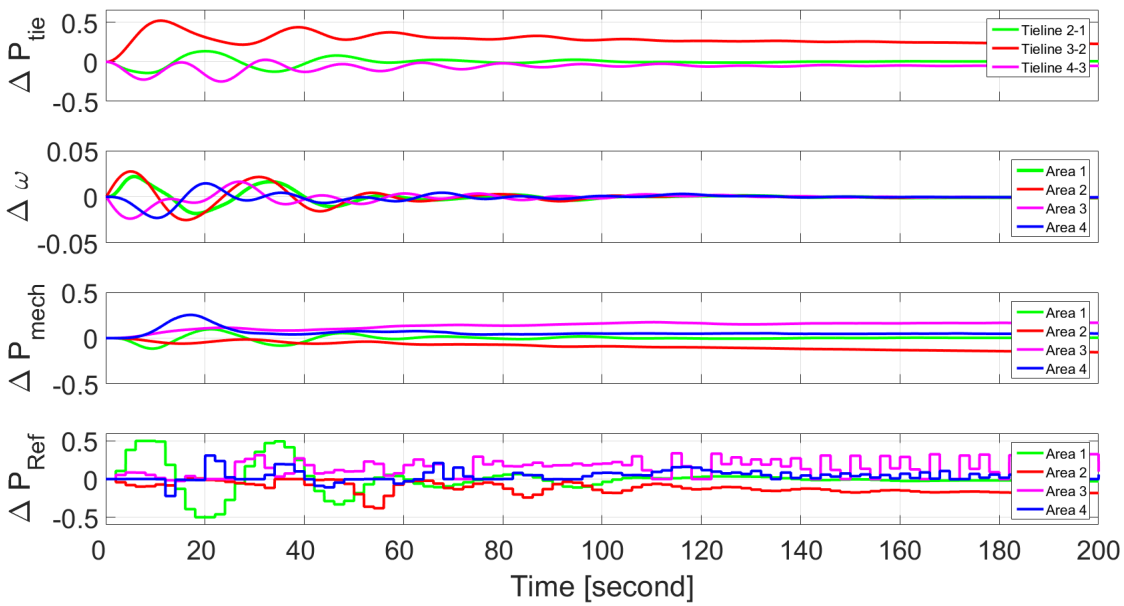

(a) Case study 3 - Partial cooperative states. The steady states are also reached after 140 seconds, approximately. The communication links to and from both the control areas no. 2 and 3 are missing. The tie-line power steady states are larger than those with full cooperative states in case study $2 \mathrm{a}$, but still smaller than those in DeMPC shown in Figure 5.
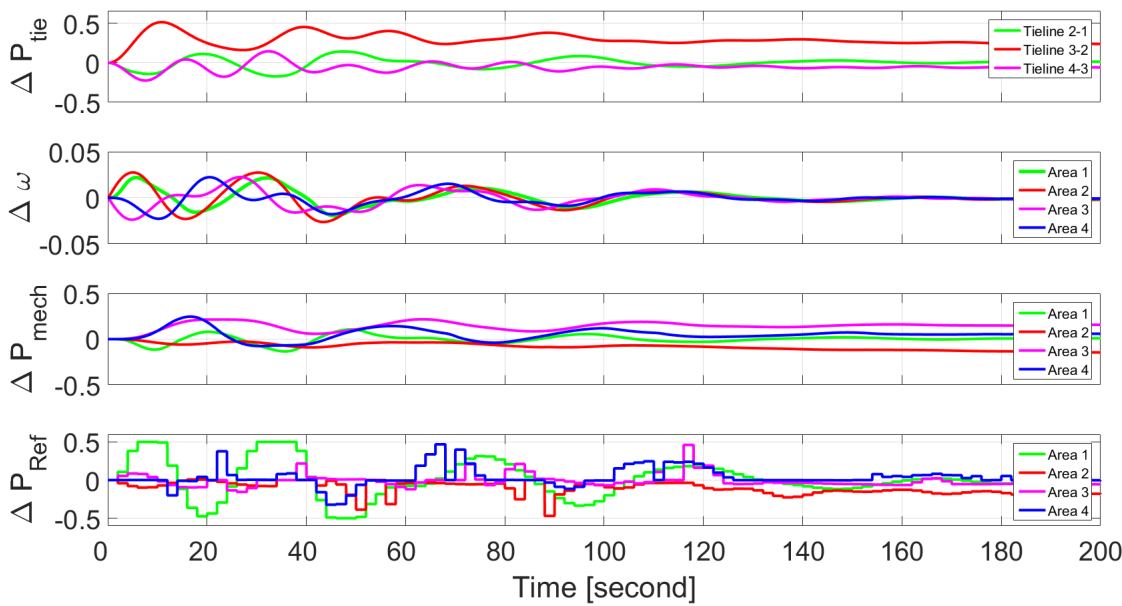

(b) Case study 4 - Partial cooperative states. The steady states are only reached after 170 seconds, approximately. Only the communication links to and from both the control area no. 2 are missing. The tie-line power steady states are larger than those with full cooperative states in case study $2 a$, but still smaller than those in DeMPC in Figure 5. The control moves are, nevertheless, smaller than those in Case 3 above.

Figure 7. Decentralised MPC with PSF and stabilising agent, shorter predictive horizon $\mathrm{N}=4$ - Two case studies of partial cooperative states. The tie-line power steady states are smaller than those in the DeMPC without any cooperative states shown in Figure 5. 


\subsection{Simulation results with the PSF strategy in an intermittent data lost environment}

The system becomes unstable with a centralised MPC without using SA in the presence of intermittent data losses with $\mu=7$. The unstable trajectory is not printed out here. The data lost process of $\mu=7$ has been simulated with random-walk variables.

Case studies 5a, 5b, and 5c:

Decentralised MPC with PSF and SA in the presence of intermittent data losses. Case study 5 a corresponds to case study 1a with full cooperative states. Case study $5 \mathrm{~b}$ corresponds to case study 3 with partial cooperative states. Case study $5 \mathrm{c}$ also corresponds to case study 3 but with a different data lost sample.

The time responses from case study 5 a are shown in Figures 8-(a) and -(b). The time responses from case study 5b are shown in Figures 9-(a) and -(b). The time responses from case study $5 \mathrm{c}$ are shown in Figures 10-(a) and -(b).

The performances in both case studies 3 and 4 in Figures 8, 9, and 10 are briefly summarised as follows: The system is stable in these three case studies thanks to the algorithm of SA in Procedure 1. The settling times, however, become longer. Notably, the tie-line power steady states are not too much different compared to those in case studies 3 and 4 without data losses.

The most relevant result here is that without SA, the system is unstable in all cases of full and partial cooperative states, also in centralised MPC, when the data are lost intermittently with $\mu=7$. 

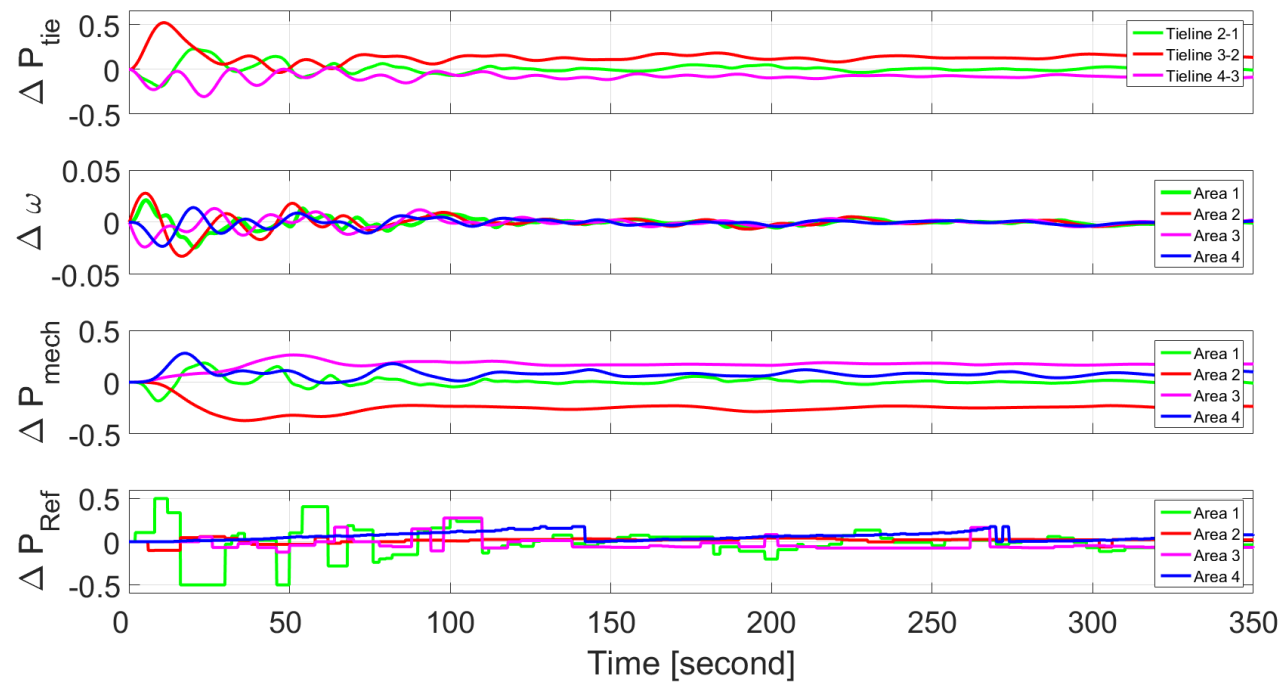

(a) The control and three states

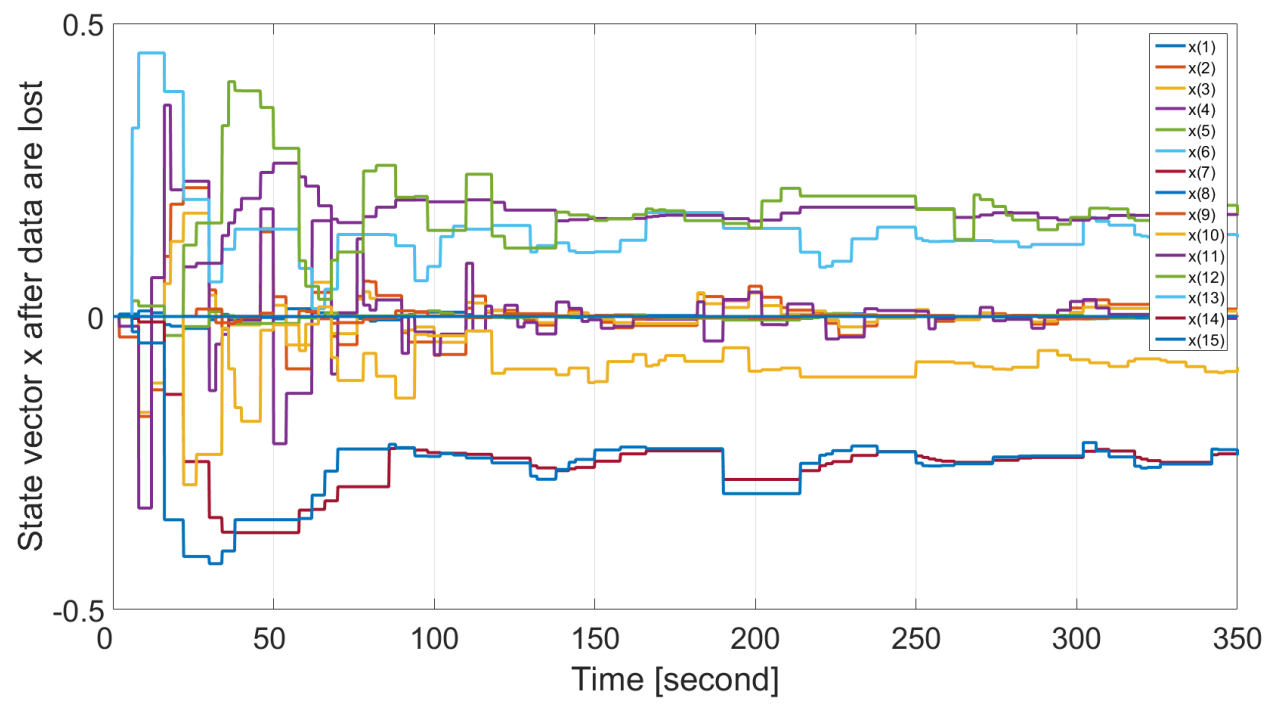

(b) All elements of the global state vector when the data are lost intermittently, $\mu=7$.

Figure 8. Decentralised MPC with PSF and stabilising agent. Case study 5a - Full cooperative states with intermittent data losses. The system remains attractive. 

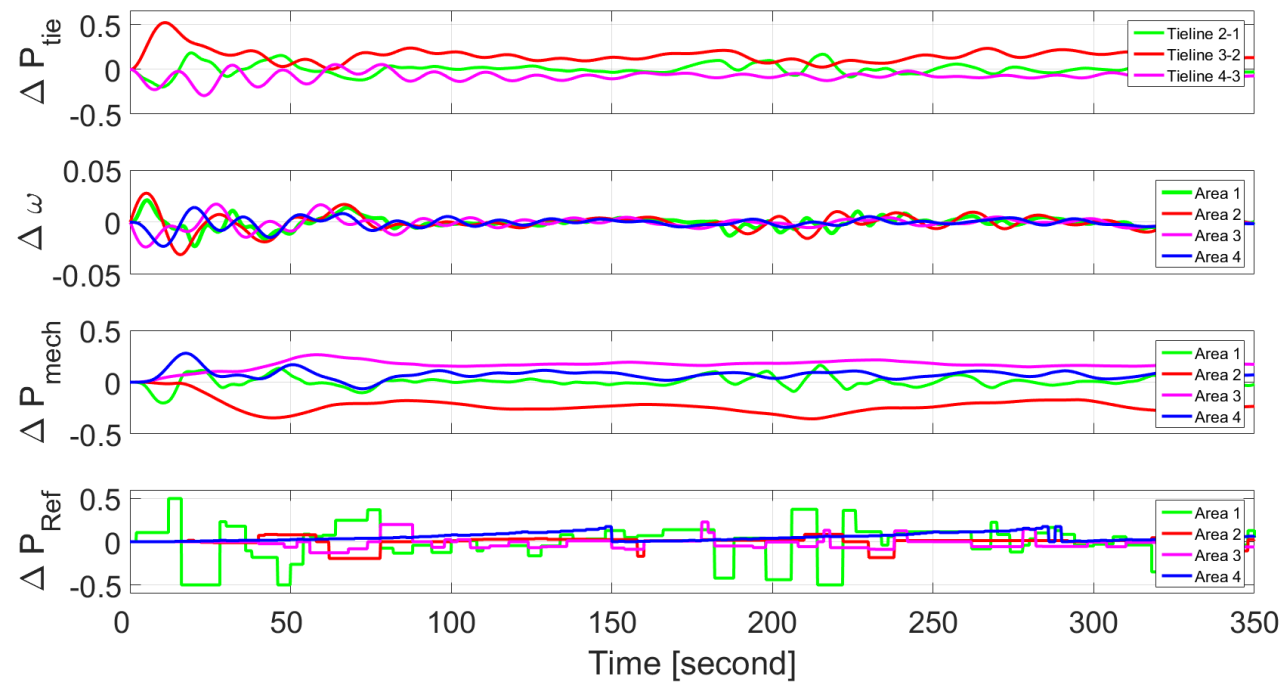

(a) The control and three states

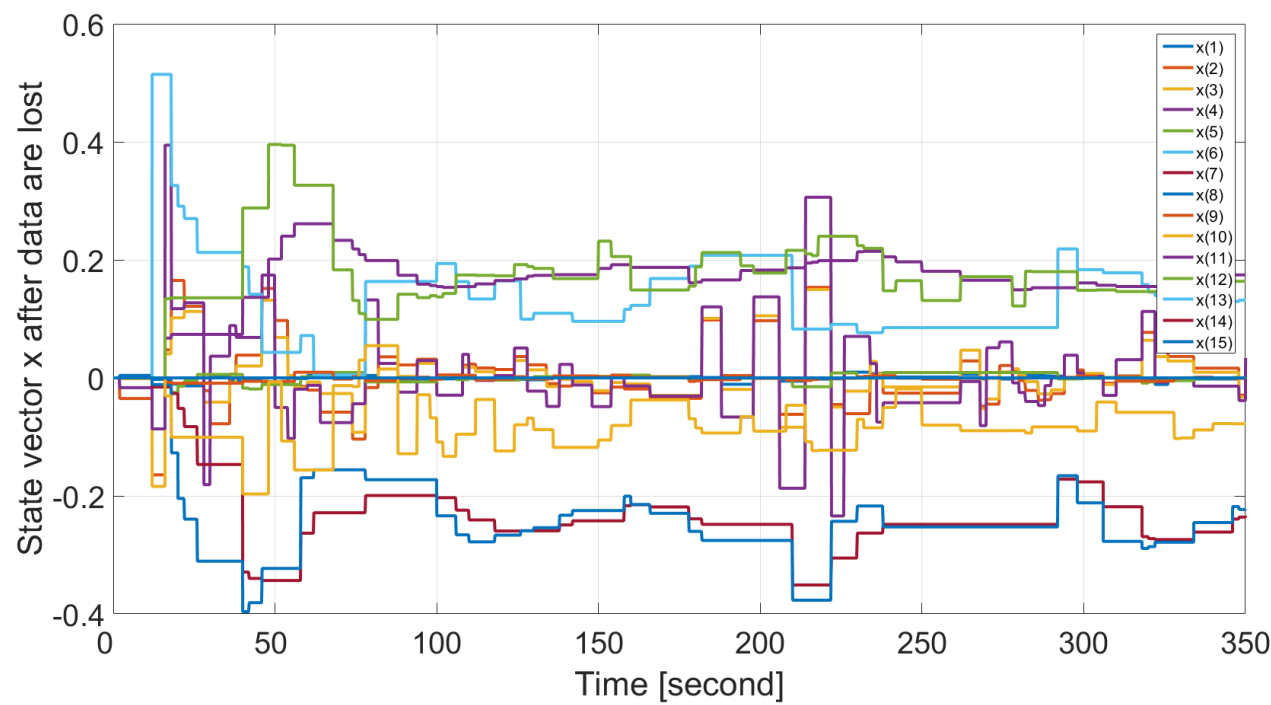

(b) All elements of the global state vector when the data are lost intermittently, $\mu=7$.

Figure 9. Decentralised MPC with PSF and stabilising agent. Case study 5b - Partial cooperative states with intermittent data losses. The system remains attractive. 

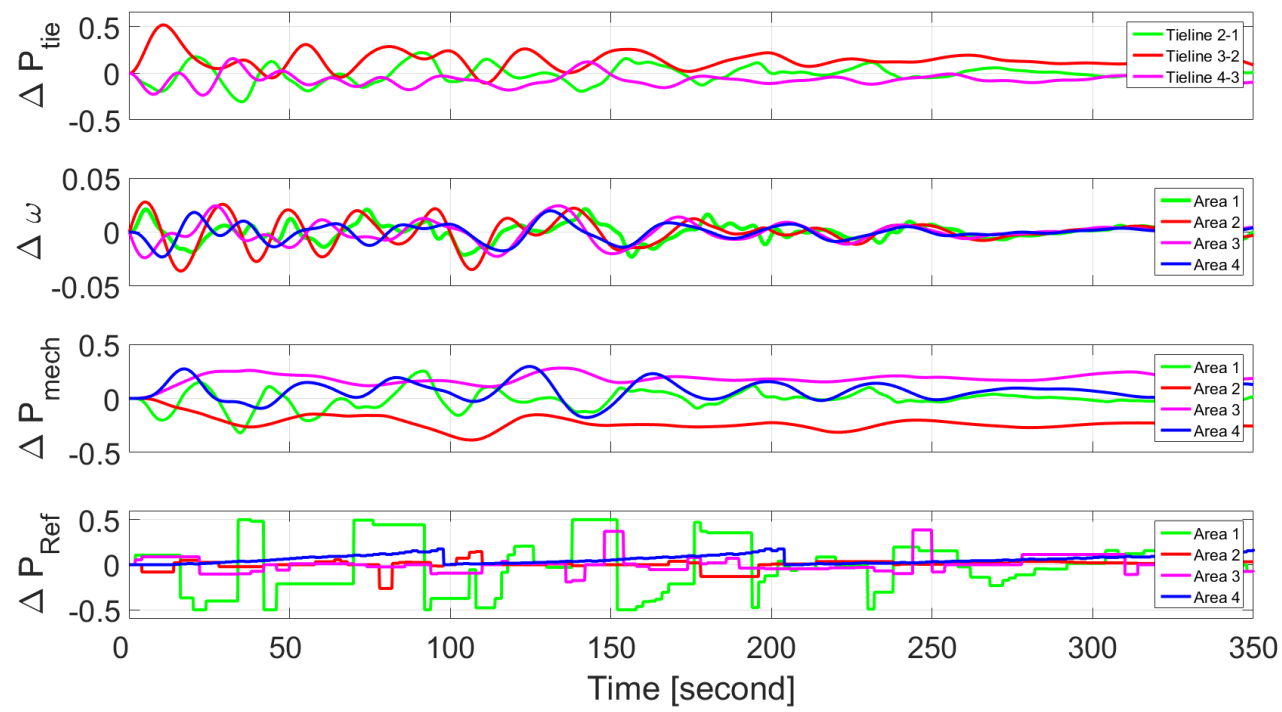

(a) The control and three states

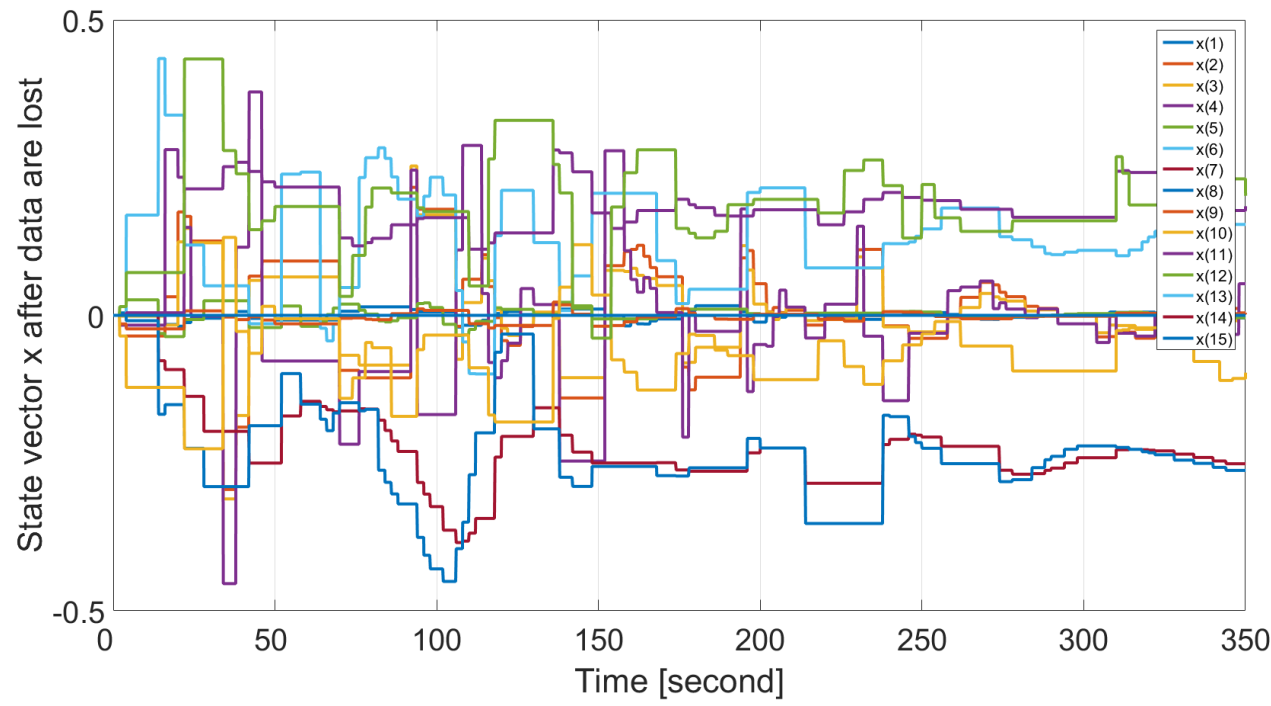

(b) All elements of the global state vector when the data are lost intermittently, $\mu=7$.

Figure 10. Decentralised MPC with PSF and stabilising agent. Case study $5 \mathrm{c}-$ Partial cooperative states with intermittent data losses. The system remains attractive.

\section{Conclusion}

A partially decentralised scheme for off-line and online computations of the semiautomatic generation control for power systems has been presented. By applying the stabilising agents in an open-loop setup, the semi-automatic control that is involved with remote human operators and local automatic control systems becomes implementable. In this approach, intermittent data losses are treated with the model-based predictions and the data-lost robust dissipativity of the controlled system. The incorporated perturbed cooperative state feedback strategy effectively reduces the demands 
on the communication links and online data by adopting the static cooperative state feedback gains and the local optimising control variables. This incorporation has resulted in the decoupled objective function for the local MPC. Not only simplifying the optimisation and using less online data, the approach offers some advantages of a semi-automatic control paradigm in an imperfect data environment. Hereby, the fusion between cooperative controls and human-machine interfaces is realisable. From the control perspective, a relaxed non-monotonic Lyapunov function has been employed in the stabilizability condition to make the decentralised control design less conservative.

\section{Acknowledgement}

We would like to thanks Dr. Robert Hunjet for thoroughly reading the manuscript and giving comments.

\section{References}

Brogliato, B., Lozano, R., Maschke, B., \& Egeland, O. (2006). Dissipative systems analysis and control: Theory and applications. Springer.

Chen, G., Yang, Y., \& Li, J. (2019). On input-to-state stability of discrete-time switched nonlinear time-varying systems. IEEE Transactions on Automatic Control, Early Access, DOI: 10.1109/TAC.2019.2910951.

Kerrigan, E. C., \& Maciejowski, J. M. (2000). Invariant sets for constrained nonlinear dicretetime systems with application to feasibility in model predictive control. Proceedings of the $39^{\text {th }}$ IEEE Conference on Decision and Control, Sydney, 4951-4956.

Kothare, M. V., Balakrishnan, V., \& Morari, M. (1996). Robust constrained model predictive control using linear matrix inequalities. Automatica, 32(10), 1361-1379.

Linsenmayer, S., Dimarogonas, D. V., \& Allgöwer, F. (2019). Periodic event-triggered control for networked control systems based on non-monotonic Lyapunov functions. Automatica, $106,35-46$.

Lunze, J. (1992). Feedback control of large scale systems. Prentice Hall.

Maciejowski, J. M. (2002). Predictive control with constraints. Prentice Hall.

Mayne, D. Q., Rawlings, J. B., Rao, C. V., \& Scokaert, P. O. M. (2000). Constraint predictive control: Stability and optimality. Automatica, 36, 789-814.

Michel, A. N., Hou, L., \& Liu, D. (2015). Stability of dynamical systems - on the role of monotonic and non-monotonic lyapunov functions, $2^{\text {nd }}$ edition. Birkhäuser.

Tran, T., \& Ha, Q. P. (2018a). Perturbed cooperative-state feedback strategy for model predictive networked control of interconnected systems. ISA Transactions, 72, 110-121.

Tran, T., \& Ha, Q. P. (2018b). A quadratic constraint approach to model predictive control of interconnected systems. Singapore: Springer Nature.

Tran, T., Maciejowski, J., \& Ling, K.-V. (2019). A general dissipativity constraint for feedback system design, with emphasis on MPC. International Journal of Robust and Nonlinear Control, Early Acess, DOI: 10.1002/RNC.4651.

Tran, T., Tuan, H. D., Ha, Q. P., \& Nguyen, H. T. (2011). Stabilising agent design for the control of interconnected systems. International Journal of Control, 84 (6), 1140-1156.

Weitenberg, E., Jiang, Y., Zhao, C., Mallada, E., Persis, C. D., \& Dörfler, F. (2018). Robust decentralized secondary frequency control in power systems: Merits and trade-offs. IEEE Transactions on Automatic Control, Early Access, DOI: 10.1109/TAC.2018.2884650.

Wood, A. J., Woolenberg, B. F., \& Sheblé, G. B. (2013). Power generation operation and control, $3^{\text {rd }}$ edition. New Jersey: John Wiley \& Son. 
Yu, Q., \& Wu, B. (2017). Generalized Lyapunov function theorems and its applications in switched systems. Systems and Control Letters, 77, 40-45.

\section{Appendices}

\section{Appendix A. State Realization Matrices, Gains and QDC Coefficient Matrices}

The global model of the four control-area AGC problem is as follows:

$$
\begin{aligned}
& A=\left[\begin{array}{llllllllll}
-0.75 & -.025 & 0.25 & 0 & 0 & 0 & 0 & 0 & 0 & 0 \\
2.54 & 0 & 0 & 0 & -2.54 & 0 & 0 & 0 & 0 & 0 \\
0 & 0 & -0.2 & 0.2 & 0 & 0 & 0 & 0 & 0 & 0 \\
-8.33 & 0 & 0 & -0.25 & 0 & 0 & 0 & 0 & 0 & 0 \\
0 & 0.025 & 0 & 0 & -.06875 & -0.025 & 0.025 & 0 & 0 & 0 \\
0 & 0 & 0 & 0 & 1.5 & 0 & 0 & 0 & -1.5 & 0 \\
0 & 0 & 0 & 0 & 0 & 0 & -0.1 & 0.1 & 0 & 0 \\
0 & 0 & 0 & 0 & -0.5714 & 0 & 0 & -0.04 & 0 & 0 \\
0 & 0 & 0 & 0 & 0 & 0.01 & 0 & 0 & -0.0571 & -0.0286 \\
0 & 0 & 0 & 0 & 0 & 0 & 0 & 0 & 2.5 & 0 \\
0 & 0 & 0 & 0 & 0 & 0 & 0 & 0 & 0 & 0 \\
0 & 0 & 0 & 0 & 0 & 0 & 0 & 0 & -1.667 & 0 \\
0 & 0 & 0 & 0 & 0 & 0 & 0 & 0 & 0 & 0.025 \\
0 & 0 & 0 & 0 & 0 & 0 & 0 & 0 & 0 & 0 \\
0 & 0 & 0 & 0 & 0 & 0 & 0 & 0 & 0 & 0 \\
0 & 0 & 0 & 0 & 0 & 0 & 0 & 0 & 0 & 0
\end{array}\right. \\
& \left.\begin{array}{lllll}
0 & 0 & 0 & 0 & 0 \\
0 & 0 & 0 & 0 & 0 \\
0 & 0 & 0 & 0 & 0 \\
0 & 0 & 0 & 0 & 0 \\
0 & 0 & 0 & 0 & 0 \\
0 & 0 & 0 & 0 & 0 \\
0 & 0 & 0 & 0 & 0 \\
0 & 0 & 0 & 0 & 0 \\
0.0285 & 0 & 0 & 0 & 0 \\
0 & 0 & -2.5 & 0 & 0 \\
-0.05 & 0.05 & 0 & 0 & 0 \\
0 & -0.0667 & 0 & 0 & 0 \\
0 & 0 & -0.06875 & -0.025 & 0 \\
0 & 0 & 0 & -0.1 & 0.1 \\
0 & 0 & -6.667 & 0 & -0.2
\end{array}\right] \\
& B=\left[\begin{array}{cccc}
B_{11} & 0 & 0 & 0 \\
0 & B_{22} & 0 & 0 \\
0 & 0 & B_{33} & 0 \\
0 & 0 & 0 & B_{44}
\end{array}\right],
\end{aligned}
$$

where $B_{11}=\left[\begin{array}{llll}0 & 0 & 0 & .25\end{array}\right]^{T} ; B_{22}=\left[\begin{array}{llll}0 & 0 & 0 & .04\end{array}\right]^{T} ; \quad B_{33}=\left[\begin{array}{llll}0 & 0 & 0 & 0.0667\end{array}\right]^{T} ; \quad B_{44}=\left[\begin{array}{llll}0 & 0 & 0 & .2\end{array}\right]^{T}$. 
The QDC coefficient matrices when $G_{c}=G_{s}$ :

$$
\begin{gathered}
R_{1}=-0.0755, Q_{1}=\left[\begin{array}{cccc}
-10.1187 & 3.1033 & -3.5984 & 0.8876 \\
3.1033 & -1.6280 & 1.2583 & -0.7481 \\
-3.5984 & 1.2583 & -2.1875 & 0.1233 \\
0.8876 & -0.7481 & 0.1233 & -0.6123
\end{array}\right], S_{1}=\left[\begin{array}{c}
0.7706 \\
-0.3267 \\
0.3128 \\
-0.0881
\end{array}\right], \\
R_{2}=-0.0704, Q_{2}=\left[\begin{array}{cccc}
-8.0863 & -0.6573 & -0.1680 & 0.6735 \\
-0.6573 & -0.9972 & -0.0167 & -0.0166 \\
-0.1680 & -0.0167 & -0.6755 & -0.1246 \\
0.6735 & -0.0166 & -0.1246 & -0.8771
\end{array}\right], S_{2}=\left[\begin{array}{c}
0.0567 \\
-0.0002 \\
-0.0047 \\
-0.0750
\end{array}\right], \\
R_{3}=-0.0714, Q_{3}=\left[\begin{array}{cccc}
-18.6982 & -1.7467 & -0.4830 & 2.2475 \\
-1.7467 & -0.9403 & -0.0510 & -0.0125 \\
-0.4830 & -0.0510 & -0.8342 & -0.0100 \\
2.2475 & -0.0125 & -0.0100 & -0.7751
\end{array}\right], S_{3}=\left[\begin{array}{c}
0.3216 \\
0.0008 \\
0.0041 \\
-0.1325
\end{array}\right], \\
R_{4}=-0.0809, Q_{4}=\left[\begin{array}{cccc}
-77.0974 & -1.4406 & 4.7235 \\
-1.4406 & -0.7103 & -0.0098 \\
4.7235 & -0.0098 & -0.6141
\end{array}\right], S_{4}=\left[\begin{array}{c}
2.3260 \\
0.0474 \\
-0.2192
\end{array}\right] .
\end{gathered}
$$

The cooperative state feedback gains when $G_{c}=G_{s}$ :

$$
\begin{aligned}
& K_{12}=10^{-13} \times\left[\begin{array}{llll}
0 & -2.747 & 0 & 0
\end{array}\right], \\
& K_{21}=\left[\begin{array}{llll}
0 & -4.8246 & 0 & 0
\end{array}\right], K_{23}=\left[\begin{array}{llll}
-48.677 & -4.795 \times 10^{-12} & 0 & 0
\end{array}\right] \text {, } \\
& K_{32}=\left[\begin{array}{llll}
-6.961 \times 10^{-12} & -4.8024 & 0 & 0
\end{array}\right], K_{34}=\left[\begin{array}{llll}
-5.809 & 0 & 0
\end{array}\right] \text {, } \\
& K_{43}=10^{-12} \times\left[\begin{array}{lll}
-0.3096 & 0 & 0
\end{array}\right] \text {. }
\end{aligned}
$$

The structures of $K_{i j}$ have been declared for the variable prior to forming the LMIs.

\section{Appendix B. Proofs}

Proof. Lemma 3.1:

From the conditions (2), the following inequality is obtained for all $k>0$ :

$$
\begin{aligned}
V(x(k)) & \leqslant \sigma V(x(k-1))+|\xi(x(k), u(k))| \\
& \leqslant \sigma[\sigma V(x(k-2))+|\xi(x(k-1), u(k-1))|]+|\xi(x(k), u(k))| \\
& \leqslant \sigma^{2} V(x(k-2))+[\sigma|\xi(x(k-1), u(k-1))|+|\xi(x(k), u(k))|] .
\end{aligned}
$$

Continuing in this way, we get

$$
V(x(k)) \leqslant \sigma^{k} V(x(0))+\sum_{\ell=0}^{k-1} \sigma^{\ell}|\xi(x(k-\ell), u(k-\ell))| .
$$

Since $|\xi(x(k), u(k))| \rightarrow 0$ as $k \rightarrow \infty$ due to the condition (3), applying the convolution sum to the second term on the right hand side of (B2), we have both the first and 
second terms on the right hand side of (B2) goes to zero as $k \rightarrow \infty$ with $0<\sigma<1$. A detailed proof for this convergence can be found in (Tran et al., 2019). Accordingly, with the condition (1) we conclude that $x(k)$ is finite for all $k>0$ and $\|x(k)\| \rightarrow 0$ as $k \rightarrow 0$.

Proof. Theorem 3.3:

Attractivity: (i) - With the conditions (1) and (4), the MPC optimisation (12) is recursively feasible with each $x_{i}(0) \in \mathbb{X}_{i}$, see, e.g., (Kerrigan \& Maciejowski, 2000). (ii) - With the conditions (2) and (3), the nominal controlled system $\Sigma$ with the PSF (5) is dissipative w.r.t. $h$ supply rates $-\xi\left(x_{\triangle i}, w_{\triangle i}\right), i=1,2, \ldots, h$, as per Definition 2.2 (with $\bar{x}=\mathcal{B} \bar{w}$ ) and the QDCs (8) of all subsystems are fulfilled for all $k>0$. We thus obtain from Lemma 3.1 that for each $x_{i}(0) \in \mathbb{X}_{i}, i=1,2, \ldots, h, x(k)$ remains bounded and $x(k) \rightarrow 0$ as $k \rightarrow \infty$.

Stability: For the global system $\Sigma$, let $\varepsilon>0$ be such that the ball of $\mathbb{B}_{\varepsilon}=\{x \mid\|x\|<\varepsilon\}$ is a subset of $\mathbb{X}: \mathbb{B}_{\varepsilon} \subset \mathbb{X}, \mathbb{X}$ is compact $\left(x(k) \in \mathbb{X} \subset \mathbb{R}^{n}\right)$. Assume $\exists\|x(k)\| \leqslant \delta_{1} \Rightarrow V(x(k)) \leqslant \mu_{1}$ and $\left\{x \mid V(x) \leqslant \mu_{1}\right\} \subset \mathbb{B}_{\varepsilon}$, and there is $\mu_{2} \geq \mu_{1}$ such that $\exists\|x(k)\| \leqslant \delta_{2} \Rightarrow V(x(k)) \leqslant \mu_{2}$ and $\left\{x \mid V(x) \leqslant \mu_{2}\right\} \subset \mathbb{B}_{\varepsilon}$, and in addition, $\left\{x \mid V(x) \leqslant \mu_{1}\right\} \subset\left\{x \mid V(x) \leqslant \mu_{2}\right\} \subset \mathbb{B}_{\varepsilon}$, with the continuous $V(x)$.

Now, suppose $\|x(0)\| \leqslant \delta_{1} \Rightarrow V(x(0)) \leqslant \mu_{1}$. From the proof of Lemma 3.1 above, we obtain

$V(x(k)) \leqslant \sigma^{k} \mu_{1}+\beta^{k-1}\left|\xi_{0}\right| \pi_{\sigma}$, where $\pi_{\sigma}>0$ is a constant depending on $\sigma$, $\xi_{0}:=\xi(x(0), u(0))$,

or $V(x(k)) \leqslant \sigma^{k} \mu_{1}+\sigma^{k-1}\left|\xi_{0}\right| \pi_{\beta}$, where $\pi_{\beta}>0$ is a constant depending on $\beta$.

Then, there always exist a time $\kappa>0$ such that $V(x(\kappa)) \leqslant \mu_{2}$, due to

(i) $\sigma^{k} \mu_{1}+\left|\xi_{0}\right| \max \left(\beta^{k-1} \pi_{\sigma}, \sigma^{k-1} \pi_{\beta}\right)<\mu_{2}$, with $\sigma<1$ and $\beta<1$, and

(ii) the assumption on the QDC feasibility $0 \geqslant \xi(x(k), u(k)) \geqslant \beta \xi(x(k-1), u(k-1))$.

Therefore, there exits $\delta_{2} \geq \delta_{1}$ such that $\|x(0)\| \leqslant \delta_{2} \Rightarrow\|x(k)\| \leqslant \varepsilon \forall k>k^{*}>\kappa$.

This is different to the Lyapunov stability in that, the above is only achieved $\forall k>k^{*}>\kappa>0$, instead of $\forall k>0$ and the storage function $V(x(k))$ of the closed-loop system is a relaxed non-monotonic Lyapunov function.

This theorem has stated that the system $\Sigma(5)$ is stabilised in the sense that $x(k)$ remains bounded and $x(k) \rightarrow 0$ as $k \rightarrow \infty$, but not asymptotically stabilised in the Lyapunov sense, similarly to the proof in (Tran et al., 2019). The proof is complete.

Proof. Theorem 3.4:

In brief, the proof is similar that for Theorem 3.3 with the addition of $\epsilon_{i} \theta_{i}$.

Denote $\tau:=\lambda_{\max }(\breve{Z})$. From the data-lost robust dissipation inequality (11) in the 
condition (3) and $d^{T}(k) d(k) \leqslant \theta$, the following inequality is obtained for all $k>0$ :

$$
\begin{aligned}
V\left(x\left(j_{q}\right)\right) & \leqslant \sigma V\left(x\left(j_{q-1}\right)\right)+\left|\xi\left(x\left(j_{q}\right), u\left(j_{q}\right)\right)\right|+\tau \theta \\
& \leqslant \sigma\left[\sigma V\left(x\left(j_{q-2}\right)\right)+\left|\xi\left(x\left(j_{q-1}\right), u\left(j_{q-1}\right)\right)\right|+\tau \theta\right]+\left|\xi\left(x\left(j_{q}\right), u\left(j_{q}\right)\right)\right|+\tau \theta \\
& \leqslant \sigma^{2} V\left(x\left(j_{q-2}\right)\right)+\left[\sigma\left|\xi\left(x\left(j_{q-1}\right), u\left(j_{q-1}\right)\right)\right|+\left|\xi\left(x\left(j_{q}\right), u\left(j_{q}\right)\right)\right|\right]+(\sigma+1) \tau \theta .
\end{aligned}
$$

Continuing in this way, we get

$$
V\left(x\left(j_{q}\right)\right) \leqslant \sigma^{q} V(x(0))+\sum_{\kappa=0}^{q-1} \sigma^{\kappa}\left|\xi\left(x\left(j_{q-\kappa}\right), u\left(j_{q-\kappa}\right)\right)\right|+\tau \theta \sum_{\kappa=0}^{q-1} \sigma^{\kappa} .
$$

Apply the QDC equality (9), which is also true with the stabilising agent due to the conditions (2) and (4), to the second term on the right hand side of (B3), we obtain

$$
V\left(x\left(j_{q}\right)\right) \leqslant \sigma^{q} V(x(0))+|\xi(x(0), u(0))| \sum_{\kappa=0}^{q-1} \sigma^{\kappa} \beta^{q-\kappa}+\theta\left(\tau \sum_{\kappa=0}^{q-1} \sigma^{\kappa}+\epsilon \sum_{\kappa=0, \kappa>2}^{q-3} \sigma^{\kappa} \beta^{q-\kappa}+3 \epsilon\right)(\mathrm{B} 4)
$$

Therefore,

$$
\begin{aligned}
V\left(x\left(j_{q}\right)\right) \leqslant \sigma^{q} V(x(0))+ & |\xi(x(0), u(0))| \max \left(\beta^{q-1} \frac{1-\sigma^{q}}{1-\sigma}, \sigma^{q-1} \frac{1-\beta^{q}}{1-\beta}\right) \\
+ & \epsilon \theta \max \left(\beta^{q-3} \frac{1-\sigma^{q-2}}{1-\sigma}, \sigma^{q-3} \frac{1-\beta^{q-2}}{1-\beta}\right) \\
& +\theta\left(\tau \frac{1-\sigma^{q}}{1-\sigma}+3 \epsilon\right),
\end{aligned}
$$

in which $\sigma^{q} V(x(0)) \rightarrow 0, \sigma^{q-1} \frac{1-\beta^{q}}{1-\beta} \rightarrow 0, \beta^{q-1} \frac{1-\sigma^{q}}{1-\sigma} \rightarrow 0, \sigma^{q-3} \frac{1-\beta^{q-2}}{1-\beta} \rightarrow 0$, $\beta^{q-3} \frac{1-\sigma^{q-2}}{1-\sigma} \rightarrow 0$, as $q \rightarrow \infty$ (i.e. as $k \rightarrow \infty$ ), and $\theta\left(\tau \frac{1-\sigma^{q}}{1-\sigma}+3 \epsilon\right)$ is a finite number.

$V(x)=x^{T} P x, P \succ 0, P$ is full row rank, and (B6) $\Rightarrow\left\|x\left(j_{q}\right)\right\| \rightarrow 0$ as $j_{q} \rightarrow \infty$ if $\theta=0$.

Furthermore, with the conditions (1) and (5), the MPC optimisation (12) is recursively feasible with each $x_{i}(0) \in \mathbb{X}_{i}$, similarly to Theorem 3.3. Therefore, $x(k)$ remains bounded and $x(k)$ goes to a neighbourhood of the origin as $k \rightarrow \infty$. The result for the case of $\theta \neq 0$ is a direct result of Theorem 2 in (Tran et al., 2019). 